\title{
Performance analysis of a common-mode signal based low-complexity crosstalk cancelation scheme in vectored VDSL
}

\author{
SM Zafaruddin*, Shankar Prakriya and Surendra Prasad
}

\begin{abstract}
In this article, we propose a vectored system by using both common mode (CM) and differential mode (DM) signals in upstream VDSL. We first develop a multi-input multi-output (MIMO) CM channel by using the single-pair CM and MIMO DM channels proposed recently, and study the characteristics of the resultant CM-DM channel matrix. We then propose a low complexity receiver structure in which the CM and DM signals of each twisted-pair (TP) are combined before the application of a MIMO zero forcing (ZF) receiver. We study capacity of the proposed system, and show that the vectored CM-DM processing provides higher data-rates at longer loop-lengths. In the absence of alien crosstalk, application of the ZF receiver on the vectored CM-DM signals yields performance close to the single user bound (SUB). In the presence of alien crosstalk, we show that the vectored CM-DM processing exploits the spatial correlation of CM and DM signals and provides higher data rates than with DM processing only. Simulation results validate the analysis and demonstrate the importance of CM-DM joint processing in vectored VDSL systems.
\end{abstract}

Keywords: alien crosstalk, common-mode signal, crosstalk cancelation, diagonal dominance, digital subscriber lines (DSL), discrete multi-tone (DMT), far-end crosstalk (FEXT), twisted pairs; vectoring

\section{Introduction}

Very high-speed digital subscriber line (VDSL) systems optimize the use of available bandwidth of the telephone lines for broadband transmissions [1]. This advanced technology can support the deployment of "triple play services" by providing high data rates (potentially upto $100 \mathrm{Mbps}$ ) through copper wires. Twisted pairs (TPs) run from the central office $(\mathrm{CO})$ to the respective customer premise equipments (CPEs) in order to provide dedicated data rates for each user. The use of the higher bandwidth frequencies (upto $30 \mathrm{MHz}$ ) generates electromagnetic couplings that cause significant crosstalk among TPs, which degrades the performance of VDSL systems. The crosstalk that originates from the subscribers enjoying similar DSL service in a coordinated system is referred to as self-crosstalk [2]. In addition to the selfcrosstalk, alien crosstalk that arises from other sources (for example, radio ingress) and non-coordinated users

\footnotetext{
* Correspondence: smzafar@ee.iitd.ac.in

Department of Electrical Engineering, Indian Institute of Technology Delhi, Hauz Khas, New Delhi 110016, India
}

\section{Springer

(c) 2012 Zafaruddin et al; licensee Springer. This is an Open Access article distributed under the terms of the Creative Commons Attribution License (http://creativecommons.org/licenses/by/2.0), which permits unrestricted use, distribution, and reproduction in any medium, provided the original work is properly cited. obtained by the VDSL systems [3]. Differential-mode (DM) processing that uses voltage differences between the two wires of the TP for the signal transmission and reception is a conventional technique to reduce the interferences, including radio ingress. In spite of this, crosstalk is the major performance limiting factor for the highbandwidth VDSL systems. Another factor limiting data rates is the significant attenuation with large loop lengths, resulting in marginal data rates even when all upstream bands are used. We demonstrate in this article that use of common-mode (CM) signals can result in significant enhancement in rates in VDSL systems as compared to systems using DM-only processing. In addition, it can help deal with alien crosstalk. The additional CM signal can be obtained by using the arithmetic mean of the voltages, measured between each wire of the TP and ground [4]. It is shown in [4] that this CM signal can be readily obtained with very little extra hardware. The $\mathrm{CM}$ channel can also be realized by using an alternative 
reference point like binder sheath [5] or one of the wires in the binder [6].

It has been shown in [7] that coordinated processing of the DM signals in all TPs (referred to as vectoring) enables cancelation of the far-end crosstalk and hence results in enormous rate increase in the DSL system. As is well known, the use of discrete multi-tone (DMT) modulation in the VDSL system makes tone-wise processing of the received signals possible. In vectored systems with $N$ TPs, this results in an $N \times N$ multi-input multioutput (MIMO) system at each of the 4096 tones used. In [7], it was shown that a decision feedback equalizer (DFE) based receiver can result in rates close to the single user bound (SUB). Subsequently, it was established in [8] that a simple zero forcing (ZF) receiver at each tone can help achieve rates very close to the SUB. Recently, an iterative receiver based on the SAGE algorithm has been proposed in [9] to eliminate the channel matrix inversion requirement of the ZF receiver while achieving near-optimal performance. When alien crosstalk is present, it is clear that various linear and non-linear receivers can be readily applied after a pre-whitening filter (see $[9,10]$, and references therein). It is noted that noise is spatially correlated across TPs due to the introduction of crosstalk from an alien source [3]. When the ZF-based receiver [8] is applied after noise whitening, it suffers from a significant performance loss due to the ill-conditioning of the channel after the whitening process as discussed in [11]. Chen et al. [10] have shown that a non-linear DFE-ZF can achieve data rate higher than a ZF receiver, though with an increase in computational complexity. In our previous study [9], we have shown that the SAGE based iterative receiver can effectively cancel the alien crosstalk to achieve a higher rate (with a little extra complexity). The MMSE based receivers have been proposed in $[11,12]$ as a tradeoff between rate and complexity by eliminating the use of a whitening filter.

Computational complexity and power consumption remain the major implementation hurdles in vectored DSL systems. Linear ZF receiver requires $\mathcal{O}\left(N^{2}\right)$ realtime computation at each of the 4096 tones. Besides this, some offline computation is required to update the inverse matrix at each tone. This is very high for realtime implementation with restrictions in computational complexity and power consumption. Several partial cancelers have been suggested $[13,14]$ that selectively cancel crosstalk and tradeoff rate for reduced complexity.

As noted already, the CM channel provides an additional mode for transmission and reception of signals through the copper wires. The CM signals at the $\mathrm{CO}$ are a result of leakage of transmitted DM signals due to imbalances in the TPs. Clearly, the CM signals are highly correlated with the DM signals [15], and consist primarily of the crosstalk (from the neighboring wires) and external noises. This suggests that the CM signals can be used as a reference for estimation of the interferences. For this reason, the CM signals have been utilized to mitigate radio frequency interference (RFI) ingress (see [16] and references therein). Yeap et al. [17] and Kamkar-Parsi et al. [18] have proposed CM-based adaptive cancelers to mitigate a single wideband noise in downstream transmissions. Pierrugues et al. [19] have used a CM signal that is orthogonal to the transmitted signal to mitigate a single noise source at the CPE. The proposed scheme is effective since the correlation between CM and DM noise is close to unity. By considering a single-TP DSL system, an algorithm based on a weighted combination of CM and DM signals has been presented in [20] to cancel a single disturber. The authors have provided extensive simulations and analytical results to show that the proposed algorithm is effective when adapted in the absence of the transmitted signal. These applications of CM signal at the receiver side for interference cancelation can be translated into improved capacity in DSL systems. Explicitly, capacity analysis using $\mathrm{CM}$-aided reception has been discussed in $[4,21,22]$. It has been shown in [4] by cable measurements, and through channel capacity expressions that the CM signals can provide significant capacity improvements, especially at high signal-to-noise ratio (SNR) levels, and low CM noise power. However, the capacity drops significantly with an increase in the number of disturbers [21]. This observation has been made rigorous in [22]. Specifically, Magesacher et al. [22] have investigated the capacity of the $\mathrm{CM}$-aided reception by considering a single DSL user. They have shown that the exploitation of CM signal can result in doubling of capacity than that possible by using DM signals alone. Note that these works have focused on cancelation of a single disturber only, by considering a non-vectored DSL system. To further enhance the performance by canceling a large number of disturbers, $\mathrm{CM}$-aided receive processing combined with vectored transmissions is well motivated for the considered system [22]. Recently, transmission of signals in the CM channel along with the co-reception of CM signals has also been studied in [5].

In this article, we propose a CM-DM system model by vectoring the co-received $\mathrm{CM}$ and $\mathrm{DM}$ signals for upstream transmissions at the $\mathrm{CO}$. We first employ a simple low complexity receiver which linearly combines the $\mathrm{CM}$ and DM signals on each TP (so as to maximize SNR for each user) and then use the signal-level coordination for the resultant signals. Since the size of the resultant vectored model remains equal to that of the DM model, the crosstalk cancelation algorithms developed for the DM system can be readily applied on the proposed CM-DM 
system model. To assess performance of the existing crosstalk cancelers on the proposed system model, we study characteristics of the equivalent CM-DM channel at each tone. To do so, we model (due to non-availability of an analytical model for the CM channel) a CM vectored channel by extending the recently proposed single-TP CM channel [18], and modify it to include the statistical dispersion (using the approach for DM channels in [23]). In particular, we show that the CM-DM channel matrix is more column-wise diagonally dominant (CWDD) than the DM channel at high frequency and/or longer loop-length. This fact is useful for development of efficient algorithms for crosstalk cancelation. We also obtain the SUB of the proposed system, and show that the CM-DM processing increases reach of the VDSL service by providing higher rates at longer loop-lengths. This advantage is accrued because of the fact that attenuation in the CM channel is smaller than the attenuation in the DM channel [18]. We extend the ZF receiver of the DM system for the proposed system, derive a simple lower bound on the capacity, and show that the SUB of CM-DM system is achieved by the ZF receiver. Finally, we use CM-DM processing to exploit high correlation of $\mathrm{CM}$ and DM signals for the mitigation of alien crosstalk. We derive an upper bound on the SUB after noise whitening and show that higher data-rate is attained with the considered system. Computer simulations are presented to further demonstrate the data-rate performance of the vectored CM-DM processing in each of the upstream frequency bands.

The remainder of this article is organized as follows. In Section 2, we describe the DSL system model. In Section 3 , we discuss a model for the CM vectored channel. We present the issue of CM-DM combining in Section 4, and elaborate on its applications to the vectored CM-DM processing in Section 5. The CM signals are exploited for the alien crosstalk cancelation in Section 6. Section 7 presents computer simulations. Finally, Section 8 provides the main conclusions.

Notation: Superscripts ${ }^{(d)}$ and ${ }^{(c)}$ indicate the DM and $\mathrm{CM}$ signals/values, respectively. Vectors and matrices are boldface small and capital letters, respectively. $\mathbf{A}(:, i)$ denotes the $i$ th column of the matrix $\mathbf{A}$ and $A_{i j}$ denotes the $i j$ th element of matrix A. Further, $\operatorname{diag}(\mathbf{A})$ denotes a diagonal matrix consisting of the diagonal elements of $\mathbf{A}$. We use $\triangleq$ to denote equality by definition. The operators $\operatorname{tr}(\cdot),|\cdot|$, and $\operatorname{det}(\cdot)$ represents trace, absolute value, and determinant, respectively, while $(\cdot)^{*},(\cdot)^{T}$, and $(\cdot)^{\dagger}$ denote the conjugate, transpose, and conjugate transpose, respectively. $p(x, y)$ denotes the joint density function of random variable $X$ and $Y$.

\section{System model}

We consider $N$ VDSL users in a binder whose receivermodems are co-located at the CO. The DMT modulation used for signal transmission partitions the channel into a number of parallel subchannels, called "tones". With transmission of only DM signal over each TP, both CM and DM signals exist at the $\mathrm{CO}$. The CM counterpart actually arises due to the leakage from the original DM signal transmission via differential to common-mode conversion. We assume synchronized transmissions, and that the impulse response length of channel is not more than one longer than the cyclic prefix length. Since each DMT tone represents an independent sub-channel, the system model presented herein is applicable to any tone. Hereafter, we omit the tone index for brevity. Denoting the symbol transmitted by the $i$ th TP by $X_{i}$, the received DM and CM signals $Y_{i}^{(d)}$ and $Y_{i}^{(c)}$ are given by:

$$
\begin{aligned}
& Y_{i}^{(d)}=H_{i i}^{(d)} X_{i}+\sum_{j=1, j \neq i}^{N} H_{i j}^{(d)} X_{j}+V_{i}^{(d)}, \\
& Y_{i}^{(c)}=H_{i i}^{(c)} X_{i}+\sum_{j=1, j \neq i}^{N} H_{i j}^{(d)} X_{j}+V_{i}^{(d)},
\end{aligned}
$$

where $V_{i}^{(d)}$ is a component of the DM additive noise that includes the alien and thermal noise at the DM port. Similarly, $V_{i}^{(c)}$ is a component of the CM additive noise that includes the alien and thermal noise at the CM port. $H_{i j}^{(d)}$ and $H_{i j}^{(c)}$ denote the crosstalk coupling coefficients from the $j$ th to the $i$ th TP.

Our model in (1) and (2) is a general case of that presented in [19]. By considering downstream transmissions, Pierrugues et al. [19] have assumed that the CM signal is strongly correlated with external noise but orthogonal to the transmitted signal. As such, the CM signal receives the alien noise only and rejects $\left(X_{i}=0\right.$ in (2)) the useful signal. It is also noted that the system of Equations (1) and (2) is a special case of that in [5, (1), (2)] where the CM channel using the binder-sheath has also been used for signal transmissions. However, in our treatment here we focus on CM reception only, and avoid consideration of transmission over the CM channel. While this concept certainly holds interesting possibilities, we believe that transmission over the CM channel is still associated with a number of difficulties, and would require further maturing of associated technologies, issues and standards before their serious induction and deployment. We also note that exploiting reception of the CM signals in the upstream requires changes only in the equipment at the $\mathrm{CO}$, and not at the CPE.

As we are extracting the DM and CM signals at each $\mathrm{TP}$, the vectoring concept used previously for only DM signals [7] can be applied for the CM signals as well. 
Therefore, the signal-level coordination of $N$ DM and $N$ $\mathrm{CM}$ received data is used to obtain individual vectored models for DM and CM system as

$$
\begin{aligned}
& \mathbf{y}^{(d)}=\mathbf{H}^{(d)} \mathbf{x}+\mathbf{v}^{(d)}, \\
& \mathbf{y}^{(c)}=\mathbf{H}^{(c)} \mathbf{x}+\mathbf{v}^{(c)},
\end{aligned}
$$

$\mathbf{y}^{(d)}=\left[Y_{1}^{(d)}, Y_{2}^{(d)}, \ldots, Y_{N}^{(d)}\right]^{T}, \mathbf{y}^{(c)}=\left[Y_{1}^{(c)}, Y_{2}^{(c)}, \ldots, Y_{N}^{(c)}\right]^{T}, \mathbf{x}=\left[X_{1}, X_{2}, \ldots, X_{N}\right]^{T}, \mathbf{v}^{(d)}=$,
where
$\left[V_{1}^{(d)}, V_{2}^{(d)}, \ldots, V_{N}^{(d)}\right]^{T}$ and $\mathbf{v}^{(c)}=\left[V_{1}^{(c)}, V_{2}^{(c)}, \ldots, V_{N}^{(c)}\right]^{T}$ are $N$-dimensional vectors of received (DM and CM), transmitted, and noise samples (DM and CM), respectively. $\mathbf{H}^{(d)}$ and $\mathbf{H}^{(c)}$ are $N \times N$ matrices with $i j$ th element defined as $H_{i j}^{(d)}=\left[\mathbf{H}^{(d)}\right]_{i j}$, and $H_{i j}^{(c)}=\left[\mathbf{H}^{(c)}\right]_{i j}$. In the following section, we formulate a CM channel model $\mathbf{H}^{(c)}$ and study its CWDD property. We later use this to study properties of the combined CM-DM channel.

\section{Common-mode channel model}

The DM channel model is well established (verified through measurements) and widely adopted in literature (see [23-25], and references therein). In particular, a stochastic MIMO channel model based on Gaussian distribution is proposed in [23] that extends the conventional 99\% worst case model [2]. Similarly, crosstalk models based on beta distribution [24] and sum-of-sinusoids [25] have been suggested to characterize the crosstalk accurately. However, an analytical model for the CM channel is not well studied in the literature. In [6], a split-pair signaling (based on the multi-conductor transmission-line theory) that offers $2 N-1$ modes of transmissions in a binder of $N$ pairs is presented by considering one of the wires as a joint common reference for all other wires. Although this configuration can provide significant improvement in data rate, the presence of ingress or egress limits the potential use of split-pair (usually not twisted) signalling. A CM channel model has also been suggested in [5] by considering the metallic-sheath of the binder as a common reference. This configuration (of $2 N$ modes) is more reliable than the split-pair signalling and significantly reduces the effect of ingress and egress on the binder provided the metallic sheath is grounded properly. A CM channel has also been developed using the shield of the binder as a reference point [26]. In [27], achievable throughput using this model has been investigated in the presence of radio ingress. It is noted that no experimental data are available for the transfer functions of any of the CM channel suggested so far.

In this study, we use earth as a common reference and develop a MIMO CM channel model by incorporating the MIMO DM stochastic channel model of [23], and the single-pair model of CM channel proposed in [18]. As discussed in [23], the stochastic DM channel $\mathbf{H}^{(d)}$ is given by

$$
\mathbf{H}^{(d)}=\mathbf{H}_{\text {diag }}^{(d)}\left[\mathbf{I}+1.59 \times 10^{-10} f \sqrt{l} \mathbf{C}^{(d)}\right],
$$

where $f$ is the frequency in $\mathrm{Hz}, l$ is the loop-length in meters, $\mathbf{H}_{\text {diag }}^{(d)}=\operatorname{diag}\left(\mathbf{H}^{(d)}\right)$ is a diagonal matrix of the DM channel $\mathbf{H}^{(d)}, \mathbf{I}$ is the $N \times N$ identity matrix, and $\mathbf{C}^{(d)}$ is a zero-diagonal matrix that reflects the crosstalk coupling dispersion (and extend the 99\% crosstalk model) as per the existing standard, including the phase. The phase of elements of the matrix $\mathbf{C}^{(d)}$ is assumed to be random, and uniformly distributed [23]. The dispersion (excluding the phase) is modeled by lognormal random variables as

$$
\left[\mathbf{C}_{k}^{(d)}\right]_{i j}=10^{-0.05 X(f)},
$$

where $X(f)$ (that models the magnitude variation) is a Gaussian random variable in $\mathrm{dB}$ with mean $\mu_{\mathrm{dB}}=2.33$ $\sigma_{\mathrm{dB}}$ and variance $\sigma_{\mathrm{dB}}$. The model presented in [23] is found to perform well for a large range of crosstalk situations. Particularly, this model is valid as long as $\mu_{\mathrm{dB}}=$ $2.33 \sigma_{\mathrm{dB}}$, which is typically observed at lower frequencies. However, for higher frequencies, this pair of $\mu_{\mathrm{dB}}$ and $\sigma_{\mathrm{dB}}$ values, tends to overestimate the crosstalk. For higher frequencies, the applicable values of these quantities have been extensively measured upto $30 \mathrm{MHz}$ (and were made available to us by Conexant Systems Inc.), which have been used in the simulations/numerical values presented in Section 7.

Using the physical model of the single-pair CM channel presented in [18] along with the DM to CM leakage factor $B(f)$ of [28], we develop a CM stochastic MIMO channel model. Specifically, we model $\mathbf{H}^{(c)}$ as:

$$
\mathbf{H}^{(c)}-\mathbf{H}_{\text {diag }}^{(c)}\left[\mathbf{I}+1.59 \times 10^{-10} f \sqrt{l} \mathbf{C}^{(c)} B(f) \phi(f)\right],
$$

where $\mathbf{H}_{\text {diag }}^{(c)}=\operatorname{diag}\left(\mathbf{H}^{(c)}\right)$ is the diagonal matrix whose $i$ th element is given as $H_{i i \text {,diag }}^{(c)}=H_{\text {line }}^{(c)} / B(f)$. Here, $H_{\text {line }}^{(c)}$ is the line insertion-loss transfer function of the CM channel which can be obtained in terms of its two-port RLCG parameters. The RLCG parameters of CM channel listed in [18] are used. The CM dispersion factor $\mathbf{C}^{(c)}$ can be expressed as $\mathbf{C}^{(c)}=B(f) H_{i i}^{(d)} \mathbf{C}^{(d)}$. The frequency-dependent leakage factor $B(f)$ is an unbalance function [28] while $\varphi(f)$ is the differential to common phase function [18]. These are defined as 


$$
B(f)=\left\{\begin{array}{c}
\sqrt{10^{5}} \text { if } 0 \leq f<150 \mathrm{~Hz} \\
\sqrt{10^{5} \cdot\left(\frac{150000}{f}\right)^{1.5}} \text { if } 150 \mathrm{kHz} \leq f \leq 30 \mathrm{MHz}
\end{array}\right.
$$

and

$$
\phi(f)=e^{J}\left(\angle H_{\text {line }}^{(c)}-\angle H_{\text {line }}^{(d)}\right) .
$$

Unbalance function $B(f)$ of lines is usually a function of frequency and determines the conversion of signals from differential-mode to common-mode. $\varphi(f)$ compensates the slower line propagation of CM compared with that of the DM. It is observed from (6) that the coupling and direct path components $\mathbf{H}^{(c)}$ can be obtained from the corresponding components of DM channel $\mathbf{H}^{(d)}$ by using the leakage factor $B(f)$.

In the frequency domain, it has been shown that the DM channel exhibits a strong CWDD property $[7,8]$. This means that the crosstalk channel $H_{j i}^{(d)}$ from a disturber $i$ into a victim $j$ is always weaker than the direct channel $H_{i i}^{(d)}$. The degree of CWDD can be characterized by the parameter $\alpha^{(d)}$ of the DM channel:

$$
\alpha^{(d)}=\max _{i, j \neq i} \frac{\left|H_{j i}^{(d)}\right|}{\left|H_{i i}^{(d)}\right|} .
$$

This CWDD property $\alpha^{(d)}$ is a function of the loop length and frequency. The characteristics of the DM channel matrix are well documented, and utilized by most of the crosstalk cancelation algorithms proposed to date [7-9]. Similarly, we define the CWDD parameter $\alpha^{(c)}$ of the CM channel as

$$
\alpha^{(c)}=\max _{i, j \neq i} \frac{\left|H_{j i}^{(c)}\right|}{\left|H_{i i}^{(c)}\right|} .
$$

Using (4), and substituting $\mathbf{C}_{j i}^{(c)}=B(f) H_{i i}^{(d)} \mathbf{C}_{j i}^{(d)}$ in (6), we can find the ratio $\eta^{(c / d)}$ of CM to DM CWDD parameters as

$$
\eta^{(c / d)}=\frac{\alpha^{(c)}}{\alpha^{(d)}}=B^{2}(f)\left|H_{i i}^{(d)}\right|
$$

We observe from (11) that the CWDD characteristic of the CM channel is also dependent on frequency and loop length. In particular, the CM channel is more diagonally dominant than the DM channel at high frequencies and/ or longer loop-lengths. This is due to the fact that both $B(f)$ and $\left|H_{i i}^{(d)}\right|$ decrease with frequency while the latter also decreases with an increase in the loop length.
A proper understanding of the CWDD characteristic of the CM channel can assist in understanding the potential gains due to joint CM-DM processing. To this end, we performed computer simulations to investigate the behavior of CWDD characteristic of the CM channel, and compare it with that of the DM channel. The ratios of CWDD parameters of both channels in three upstream bandwidths over various loop lengths are plotted in Figure 1. The frequency ranges of 3.75 to $5.2 \mathrm{MHz}, 8.5$ to $12 \mathrm{MHz}$, and 18.1 to $30 \mathrm{MHz}$ are referred to as a low-frequency band (LFB), mid-frequency band (MFB) and high-frequency band (HFB), respectively. Unlike in all previous studies, we also study characteristics of the channel in the HFB, where the characteristics are quite different from those of the LFB and MFB. Figure 1a shows that the CM channel is not as CWDD as the DM channel in the LFB for shorter loop lengths. However, the CWDD property improves with an increase in frequency and loop length as shown in Figure 1b,c. The main conclusion of these studies is that the CM channel is more CWDD than the DM channel at higher frequencies and/or longer loop-lengths, a fact which is consistent with the analytical expression in (11). In the HFB, the CWDD nature of the DM matrix is quite weak as $\alpha^{(d)}$ increases with frequency and loop length. However, the variation of $\alpha^{(c)}$ with frequency and loop length is opposite to that of $\alpha^{(d)}$. Thus, the CM MIMO channel is well conditioned (in as far as the CWDD property is concerned) at a higher bandwidth and/or longer loop-length (as compared to DM MIMO channel), which motivates the use of CM signals along with DM signals for crosstalk cancelation.

\section{Single-TP CM-DM processing}

In this section, we employ CM-DM joint processing by linearly combining the CM and DM signals at each TP to improve the SNR of each user while treating all the crosstalk as interference. Vectored crosstalk cancelation scheme based on such a processing is studied in the following section. We first assume the presence of white noise only, while spatially correlated noise due to the alien crosstalk is discussed in Section 6.

To facilitate interference cancelation with a linear combination of the CM and DM signals, we obtain signals after frequency-domain equalization (FEQ) $\left(Z_{i}^{(d)}=Y_{i}^{(d)} / H_{i i}^{(d)}\right.$ and $\left.Z_{i}^{(c)}=Y_{i}^{(c)} / H_{i i}^{(c)}\right)$ as :

$$
\begin{aligned}
& Z_{i}^{(d)}=X_{i}+\sum_{j=1, j \neq i}^{N} \frac{H_{i j}^{(d)}}{H_{i i}^{(d)}} X_{j}+V_{i}^{(d)}, \\
& Z_{i}^{(c)}=X_{i}+\sum_{j=1, j \neq i}^{N} \frac{H_{i j}^{(c)}}{H_{i i}^{(c)}} X_{j}+V_{i}^{(c)},
\end{aligned}
$$




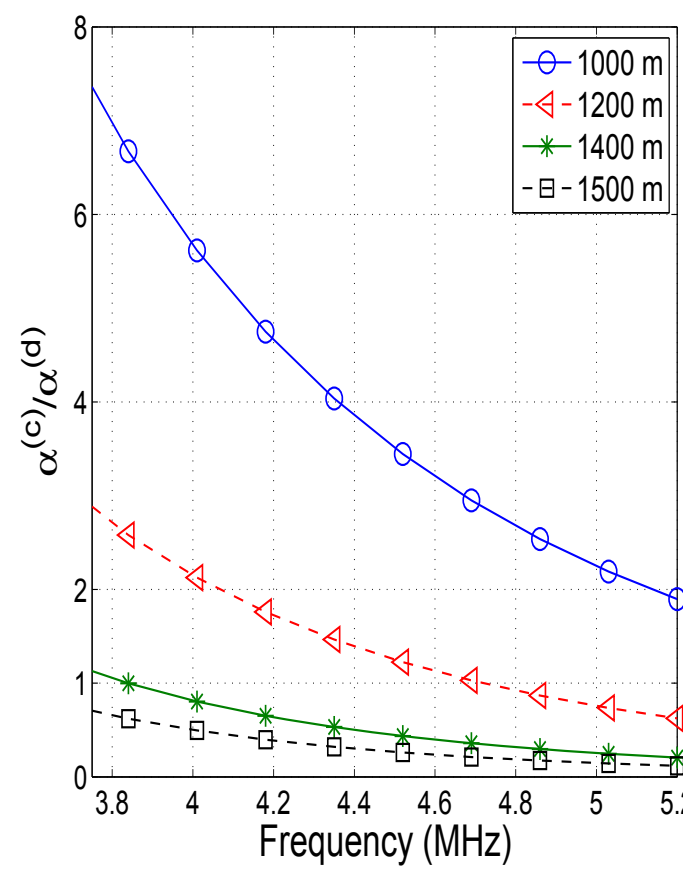

(a)

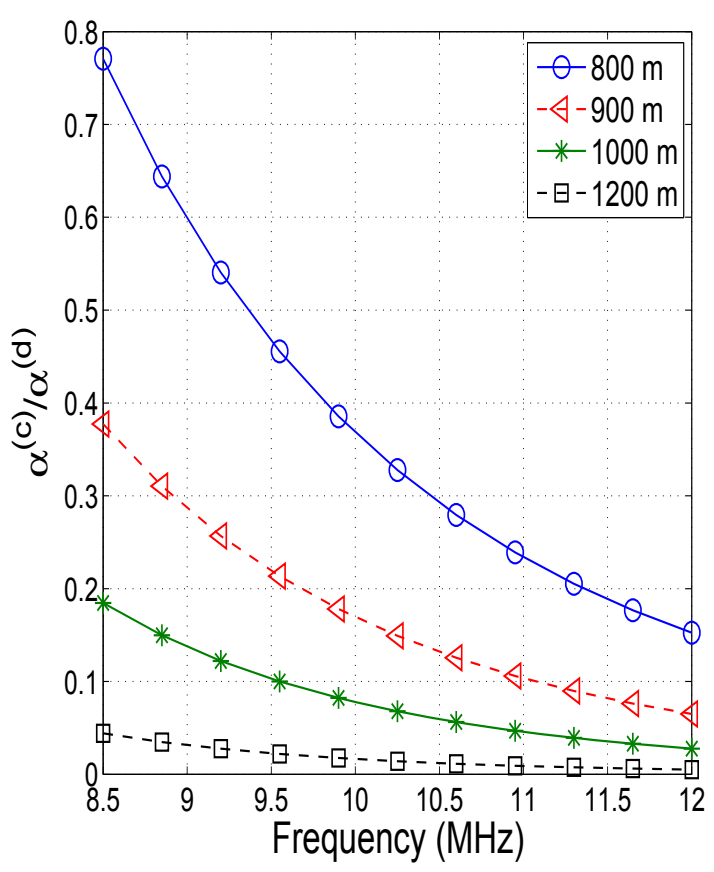

(b)

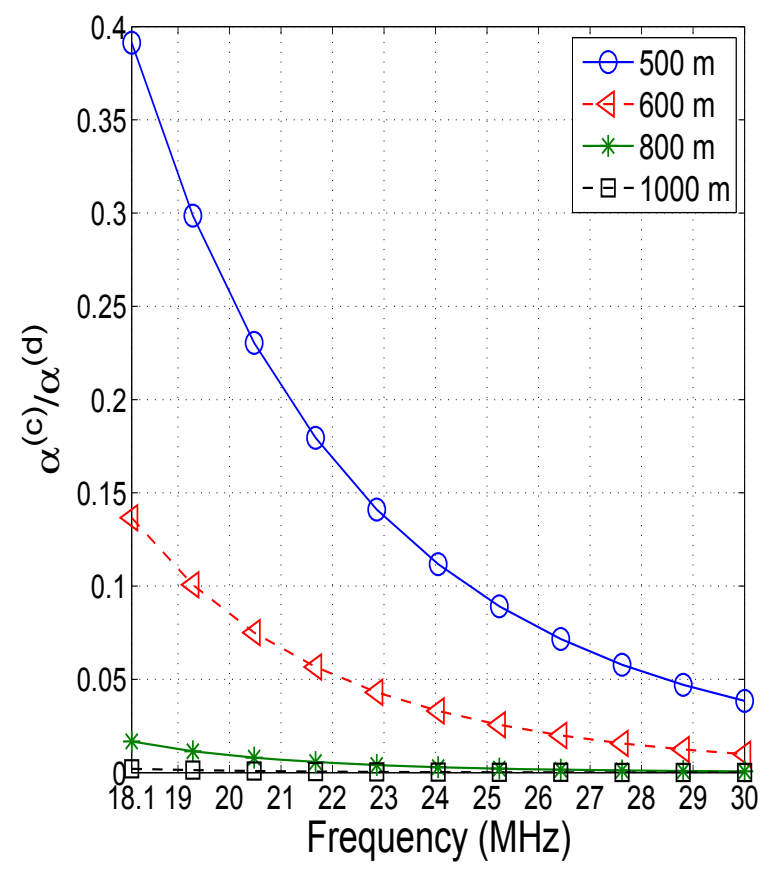

(c)

Figure 1 Ratio of CM to DM CWDD parameters at various loop lengths in all three upstream bands.

where $V_{i}^{(d)}=V_{i}^{(d)} / H_{i i}^{(d)}$, and $V_{i}^{(c)}=V_{i}^{(c)} / H_{i i}^{(c)}$ are noise terms after FEQ. We assume that the central limit theorem holds for the second term of each of the two equations in (12), so that it can be regarded as a circular symmetric Gaussian random variable. This is reasonable, since the far end crosstalk (FEXT) component consists of the weighted sum of independent symbols. With this, $\sum_{j=1, j \neq i}^{N} \frac{H_{i j}^{(d)}}{H_{i i}^{(d)}} X_{j}$ is Gaussian with mean zero and variance 
equal to $\operatorname{FEXT}_{i}^{(d)}=\sum_{j=1, j \neq i}^{N}\left|\frac{H_{i j}^{(d)}}{H_{i i}^{(d)}}\right|^{2} \sigma_{x, j}^{2}$ giving SNR without FEXT cancelation for the $i$ th user as $\mathrm{SNR}_{i, \mathrm{fext}}^{(d)}=\frac{\sigma_{x, i}^{2}}{\mathrm{FEXT}_{i}^{(d)}+\sigma_{v^{\prime}, i}^{2(d)}}$. Here, $\sigma_{v^{\prime}, i}^{2(d)}$ is the variance of the noise $V_{i}^{(d)}$ after FEQ. Similarly, the FEXT component of the CM signal has zero mean and variance $\operatorname{FEXT}_{i}^{(c)}=\sum_{j=1, j \neq i}^{N}\left|\frac{H_{i j}^{(c)}}{H_{i i}^{(c)}}\right|^{2} \sigma_{x, j}^{2}$, thus resulting in an SNR of $\mathrm{SNR}_{i, \text { fact }}^{(c)}=\frac{\sigma_{x, i}^{2}}{\mathrm{FEXT}_{i}^{(c)}+\sigma_{v^{\prime}, i}^{2(c)}}$. Similar to the DM system, $\sigma_{v^{\prime}, i}^{2(c)}$ is the variance of the noise $V_{i}^{(c)}$ after FEQ. It is noted that in the presence of a large number of disturbers with high power spectral densities (PSD), $\mathrm{SNR}_{i, \text { fact }}^{(d)}$ is quite low, necessitating the cancelation of crosstalk. When the FEXT is canceled, crosstalk-free SNR (bit loading is based on this quantity) $S_{i}^{(d)}=\frac{\sigma_{x, i}^{2}}{\sigma_{v^{\prime}, i}^{2()}}$ can be obtained. As stated earlier, we use the CM signals for the cancelation of interferences to improve the performance of the DM channel.

Define $1=[1,1]^{T}$. With this, the ML estimate $\hat{X}_{i}$ can be obtained by maximization of the likelihood function $p\left(Z_{i}^{(d)}, Z_{i}^{(c)} / X_{i}\right)$ as:

$$
\hat{X}_{i}=\left(1^{T} \mathbf{R}_{i}^{-1} 1\right)^{-1} 1^{T} \mathbf{R}_{i}^{-1} \mathbf{z}_{i}^{(c d)} .
$$

We can obtain a noise covariance matrix $\mathbf{R}_{i}$ of $\mathbf{z}_{i}^{(c d)}=\left[Z_{i}^{(d)}, Z_{i}^{(c)}\right]^{T}$ as

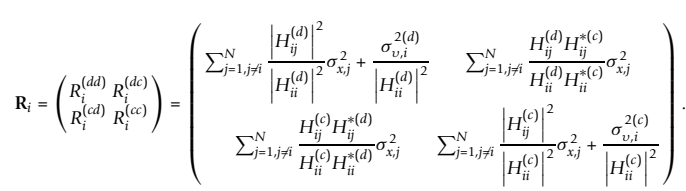

We note that the estimator in (13) bases its estimate on a weighted sum of the CM and DM signals. Therefore, we simplify (13) to get

$$
\hat{X}_{i}=\frac{\left(R_{i}^{(c c)}-R_{i}^{(c d)}\right) Z_{i}^{(c d)}}{\left(R_{i}^{(d d)} R_{i}^{(c c)}-R_{i}^{(d c)} R_{i}^{(c d)}\right)^{2}\left(R_{i}^{(d d)}+R_{i}^{(c c)}-R_{i}^{(d c)}-R_{i}^{(c d)}\right)},
$$

where the combined signal $Z_{i}^{(c d)}$ is given by:

$$
Z_{i}^{(c d)}=Z_{i}^{(d)}+K_{i} Z_{i}^{(c)}
$$

and coefficient $K_{i}$ can be obtained as

$$
K_{i}=\frac{R_{i}^{(d d)}-R_{i}^{(d c)}}{R_{i}^{(c c)}-R_{i}^{(c d)}} .
$$

By assuming equal transmit power $\sigma_{x, j}^{2}=\sigma_{x, i}^{2}$ and using (14) in (17), we can represent $K_{i}$ as

$$
\begin{aligned}
K_{i}= & \frac{S_{i}^{(c)}\left[1+S_{i}^{(d)} \sum_{j \neq i}\left(\left|\frac{H_{i j}^{(d)}}{H_{i i}^{(d)}}\right|^{2}-\frac{H_{i j}^{(d)} H_{i j}^{*(c)}}{H_{i i}^{(d)} H_{i i}^{*(c)}}\right)\right]}{S_{i}^{(d)}\left[1+S_{i}^{(c)} \sum_{j \neq i}\left(\left|\frac{H_{i j}^{(c)}}{H_{i i}^{(c)}}\right|^{2}-\frac{H_{i j}^{*(d)} H_{i j}^{(c)}}{H_{i i}^{*(d)} H_{i i}^{(c)}}\right)\right]} \\
= & \frac{\operatorname{SNR}_{i, \text { fact }}^{(c)}}{\operatorname{SNR}_{i, \text { fact }}^{(d)}}\left[\frac{1-\mathrm{SNR}_{i, \text { fact }}^{(d)} \sum_{j \neq i} \frac{H_{i j}^{(d)} H_{i j}^{*(c)}}{H_{i i}^{(d)} H_{i i}^{*(c)}}}{1-\mathrm{SNR}_{i, \text { fext }}^{(c)} \sum_{j \neq i} \frac{H_{i j}^{*(d)} H_{i j}^{(c)}}{H_{i i}^{*(d)} H_{i i}^{(c)}}}\right] .
\end{aligned}
$$

The exact value of $K_{i}$ can readily be determined with channel knowledge. However, to obtain an understanding of the SNR and CWDD behavior of the combined signal, an approximated value of $K_{i}$ can be obtained. The last term $\sum_{j \neq i} \frac{H_{i j}^{(d)} H_{i j}^{*(c)}}{H_{i i}^{(d)} H_{i i}^{*(c)}}$ in the numerator (and a similar term in the denominator) of (18) is very small for large $N$ because of small magnitudes (due to CWDD property) and the independent phase factors in $\frac{H_{i j}^{(d)}}{H_{i i}^{(d)}}$ and $\frac{H_{i j}^{(c)}}{H_{i i}^{(c)}}$. Thus, their influence on $K_{i}$ can be ignored to get

$$
K_{i} \approx \frac{\mathrm{SNR}_{i, \mathrm{fext}}^{(c)}}{\mathrm{SNR}_{i, \mathrm{fext}}^{(d)}} .
$$

It can be shown that the SNR after the CM-DM combining using $K_{i}$ as implied by (13) is always greater than the SNR that can be obtained using the DM signals alone. To do so we define: $e_{i}^{(d)}=\sum_{j=1, j \neq i}^{N} \frac{H_{i j}^{(d)}}{H_{i i}^{(d)}} X_{j}+V_{i}^{(d)}$ (the sum of FEXT and noise in the DM signal after FEQ), $e_{i}^{(c)}=\sum_{j=1, j \neq i}^{N} \frac{H_{i j}^{(c)}}{H_{i i}^{(c)}} X_{j}+V_{i}^{\prime(c)}$ (the sum of FEXT and noise in the CM signal after FEQ), and $\mathbf{e}_{i}^{(c d)}=\left[e_{i}^{(d)}, e_{i}^{(c)}\right]^{T}$. With this, we can re-write (12) as: 


$$
\mathbf{z}_{i}^{(c d)}=1 X_{i}+\mathbf{e}_{i}^{(c d)} .
$$

Now consider a Minimum Output Energy (MOE) type of receiver that takes the weighted sum of the components of $\mathbf{z}_{i}^{(c d)}$ such that $\mathbf{w}_{i}^{H} 1=1$ while minimizing the output energy $E\left\{\left|\mathbf{w}_{i}^{H} \mathbf{z}_{i}^{(c d)}\right|^{2}\right\}$. It can be shown in a straightforward manner that such a receiver is again given by (13). Since such receivers are known to have SNR maximization property, it is clear that the weighted sum of CM and DM signals implied by (13) results in an SNR that is higher than when using only DM signals. We now get an expression of SNR using joint CM-DM processing for the $i$ th user as

$$
\operatorname{SNR}_{i}^{(c d)}=\frac{\left|H_{i i}^{(c d)}\right|^{2} \sigma_{x, i}^{2}}{\sum_{j=1, j \neq i}^{N}\left|H_{i j}^{(c d)}\right|^{2} \sigma_{x, j}^{2}+\sigma_{v, i}^{2(c d)}},
$$

where $H_{i i}^{(c d)}=H_{i i}^{(d)}+K_{i} H_{i i}^{(c)}, H_{i j}^{(c d)}=H_{i j}^{(d)}+K_{i} H_{i j}^{(c)}$ and $\sigma_{v, i}^{2(c d)}$ is the variance of $V_{i}^{(d)}+K_{i} V_{i}^{(c)}$. Simulation results plotted (for a frequency tone of $12 \mathrm{MHz}$, loop length of $500 \mathrm{~m}$, and 10 users) in Figure 2 clearly shows the increase in SNR due to such CM-DM combining at each TP. We can find the capacity $\mathcal{R}_{i}^{(c d)}$ of the singleTP CM-DM processing with SNR-gap to capacity $\Gamma$ as

$$
\mathcal{R}_{i}^{(c d)}=\log _{2}\left(\operatorname{det}\left(\mathbf{I}+\Gamma^{-1} \sigma_{x, i}^{2}\left(\overline{\mathbf{R}}_{i}\right)^{-1} \overline{\mathbf{h}}_{i}^{(c d)} \overline{\mathbf{h}}_{i}^{(c d) \dagger}\right)\right),
$$

where $\overline{\mathbf{h}}_{i}^{(c d)}=\left[H_{i i}^{(d)}, H_{i i}^{(c)}\right]^{T}$, and $\overline{\mathbf{R}}_{i}$ is the covariance matrix of total noise (white noise and FEXT) before FEQ. We can deduce from Figure 2 that the joint single-TP CM-DM processing achieves capacity close to the theoretical capacity in the presence of a few crosstalker only. Similar observations have been made in

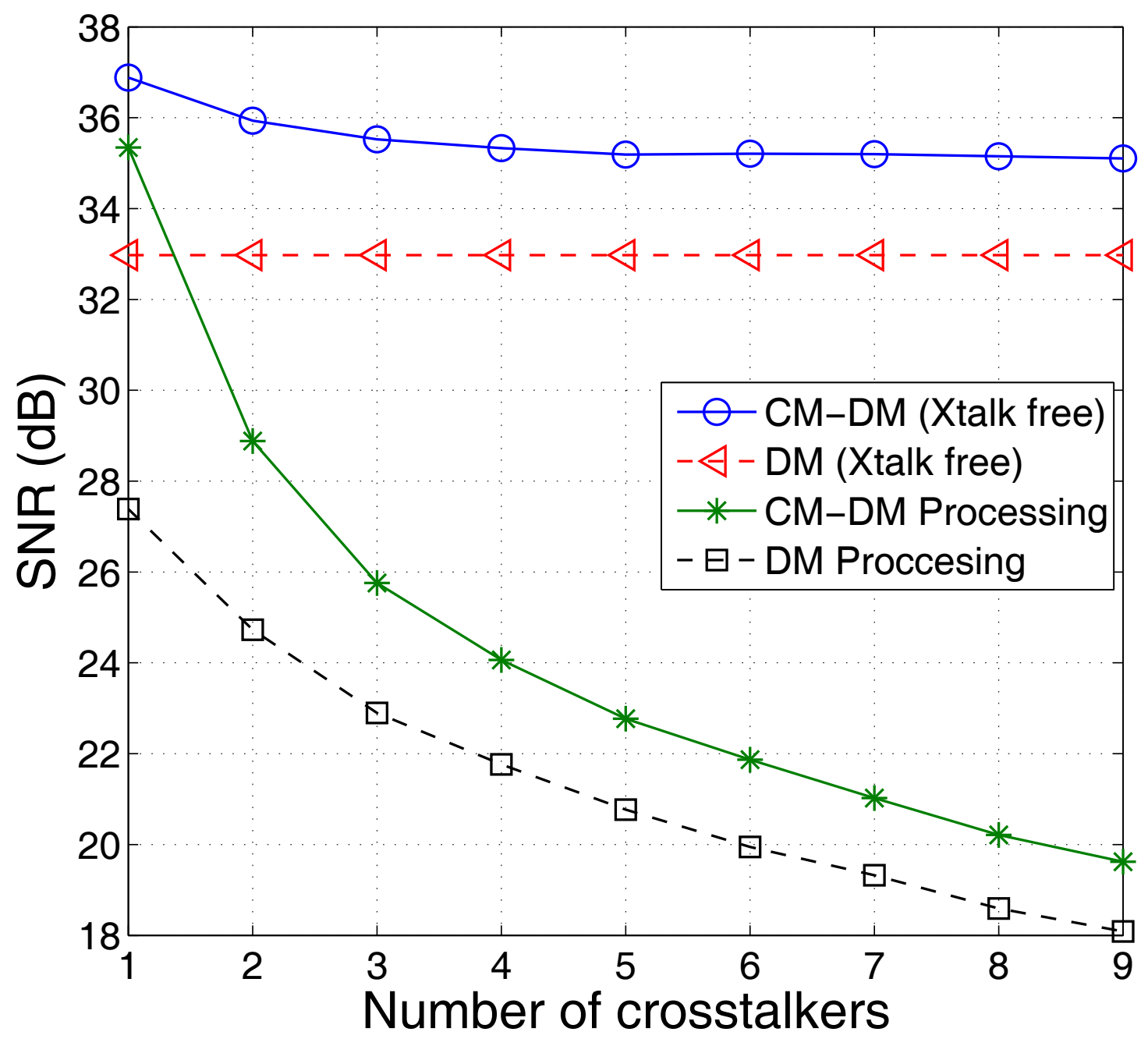

Figure 2 SNR performance of the single-TP CM-DM and DM processing with a frequency tone of $12 \mathrm{MHz}$ at a loop length of $500 \mathrm{~m}$. 
$[20,22]$. This motivates the use of vectoring of the CM signals for the cancelation of a number of disturbers.

\section{Vectored CM-DM processing}

As such, a joint signal-level coordination among TPs can be realized by concatenating $\alpha^{(d)}$ the vectored DM and $\mathrm{CM}$ signals of (3) as:

$$
\mathbf{y}^{(c+d)}=\left[\begin{array}{l}
\mathbf{y}^{(d)} \\
\mathbf{y}^{(c)}
\end{array}\right]=\mathbf{H}^{(c+d)} \mathbf{x}+\mathbf{v}^{(c+d)},
$$

where $\mathbf{y}^{(c+d)}$ is $2 N \times 1$ received vector, $\mathbf{H}^{(c+d)}=\left[\mathbf{H}^{T(d)}, \mathbf{H}^{T(c)}\right]^{T}$ is a $2 N \times N$ matrix of channel coefficients, and $\mathbf{v}^{(c+d)}=\left[\mathbf{v}^{T(d)}, \mathbf{v}^{T(c)}\right]^{T}$ is the vector of noise samples. To show the benefit of CM-aided processing, we obtain the SUB of the $2 N$-dimensional vectored CM-DM model of (23). SUB is the capacity achieved when a single user transmits and all the receivers of the vectored system are used to detect the transmitted signal via multi-paths, i. e., based on the signals received on all the TP's (direct line and crosstalk paths) [8]. The SUB of the concatenated vectored $\mathrm{CM}$ and $\mathrm{DM}$ signals can be expressed as

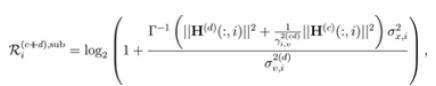

where $\gamma_{i, v}^{2(c d)}=\sigma_{i, v}^{2(c)} / \sigma_{i, v}^{2(d)}$ is the ratio of CM and DM noise variances. This ratio is generally greater than unity as CM port is usually more noisy than the DM port. We define $\beta_{i}^{(c d)}=\left|\frac{H_{i i}^{(c)}}{H_{i i}^{(d)}}\right|$ as the ratio of the frequency response of diagonal channel coefficients of the $\mathrm{CM}$ and DM channels. This ratio is greater than one when $\left|H_{i i}^{(c)}\right|>\left|H_{i i}^{(d)}\right|$, which holds at high frequency tones and/or longer loop lengths. This has also been observed in [5] for the CM channel using the binder sheath.

We use (24) and CWDD parameters of the CM and DM channels, to obtain an upper bound on the SUB of the $2 \mathrm{~N}$-dimensional CM-DM system as

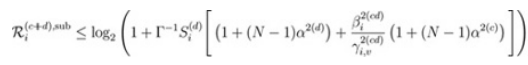

From (25), we can express the SUB with only DM processing as [8]

$$
\mathcal{R}_{i}^{(d), \text { sub }} \leq \log _{2}\left(1+\Gamma^{-1} S_{i}^{(d)}\left(1+(N-1) \alpha^{2(d)}\right)\right) .
$$

From (25) and (26), we can see that $\mathcal{R}_{i}^{(c+d) \text { sub }} \geq \mathcal{R}_{i}^{(d) \text { sub }}$ when $\underline{\beta_{i}^{2(c d)}}$ $\frac{\beta_{i}^{2(c d)}}{\gamma_{i, v}^{2(c d)}}$ is high (which occurs at high frequency and/or longer loop lengths as noted earlier). This shows that a substantial rate enhancement may be realized with the vectored CM-DM processing at longer loop-lengths.
Although the above mentioned model can effectively improve the rate performance, it imposes additional complexity in implementing the existing (ZF or DFE) receivers, since the size of the effective channel matrix $\mathrm{H}^{(++\infty)}$ is large $(2 N \times N)$. As discussed earlier, the vectored model in [5] has the resultant channel matrix of size $2 N \times 2 N$ and thus increases the complexity further for the implementation of equalizers. Therefore, instead of the step involved in (23), we formulate a new $N$-dimensional CM-DM system model by exploiting (16) for each TP and vectorizing the resultant signals as

$$
\mathbf{y}^{(\mathrm{cd})}=\mathbf{H}^{(\mathrm{cd})} \mathbf{x}+\mathbf{v}^{(\mathrm{cd})}
$$

where $i j$ th element of $\mathbf{H}^{(c d)}$ is $H_{i j}^{(d)}+K_{i} H_{i j}^{(c)}$ and the $i$ th sample of $\mathbf{v}^{(\mathbf{c d})}$ is given by $V_{i}^{(d)}+K_{i} V_{i}^{(c)}$. With such a vectoring approach, the size of effective channel $\mathbf{H}^{\text {(cd) }}$ remains the same and thereby receiver-complexity for the CM-DM system is comparable to that of the conventional, $N$-dimensional vector $\mathrm{DM}$ processing. Note that there is only one signal at each TP, so that the vectored channel matrix again becomes of size $N \times N$ at each tone. In fact, only $\mathcal{O}(N)$ multiplications are required per tone for the operation of linear combinations of the CM and DM signals. Hereafter we refer to this $N \times N$ equivalent channel as the $N$-dimensional CM-DM vectored channel at each tone. Studying this lower dimensional CM-DM joint processing in the vectoring context is important since the CM-DM combining at the TP influences the channel matrix (the CWDD parameter and the SNR), and hence the attainable rate with vectoring. It is important to understand the structure of this matrix well so that performance of the standard crosstalk cancelers on the equivalent signal after the TP level CM-DM combining can be assessed. We show later that the application of ZF canceler on $\mathrm{N}$ dimensional CM-DM vectored system results in performance close to the SUB of the same system. In what follows, we study the theoretical capacity of the considered CM-DM system by using the CWDD property of the resultant channel $\mathbf{H}^{(c d)}$.

\subsection{CWDD parameter of the CM-DM channel and SUB}

In this subsection, we show that the new $\mathrm{N}$-dimensional vectored channel $\mathbf{H}^{(c d)}$ in (27) using the combined CMDM signals is more strongly CWDD than the channel matrix with only vectored DM signals, especially at higher frequency-tones and/or longer loop-lengths. The CWDD parameter (denoted by $\alpha^{(c d)}$ of the resultant channel $\mathbf{H}^{(c d)}$ can be upper bounded in terms of CWDD parameters $\left(\alpha^{(d)}\right.$ and $\left.\alpha^{(c)}\right)$ of the individual channel as 


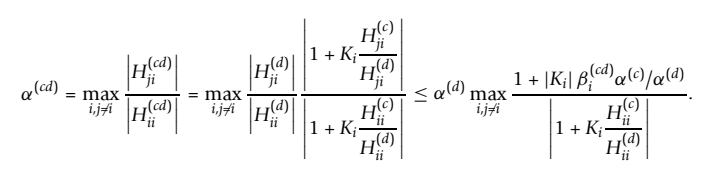

Using $\eta^{(c / d)}=\alpha^{(c)} / \alpha^{(d)}$ in (28), we can find an upper bound on the ratio $\eta^{(c d / d)}=\alpha^{(c d)} / \alpha^{(d)}$ of CM-DM to DM CWDD parameters as

$$
\eta^{(c d / d)} \leq \max _{i, j \neq i} \frac{1+\left|K_{i}\right| \beta_{i}^{(c d)} \eta^{(c / d)}}{\left|1+K_{i} \frac{H_{i i}^{(c)}}{H_{i i}^{(d)}}\right|} .
$$

It is obvious from (29) that $\eta^{(c d / d)}<1$ if $\eta^{(c / d)}<1$. Hence, we can say that if $\alpha^{(c)}<\alpha^{(d)}$, then the vectored CM-DM channel obtained after combining the CM and DM signals at each TP has a CWDD parameter that is smaller (that is, $\left.\alpha^{(c d)}<\alpha^{(d)}\right)$. We have already illustrated in Figure 1 that the condition $\eta^{(c / d)}<1$ is usually satisfied at higher frequencies and/or longer loop-lengths. We also plot the ratio of the CWDD parameters of CM-DM and DM channel in Figure 3. It can be seen from this figure that the resultant channel $\mathbf{H}^{(c d)}$ is more CWDD at higher frequencies and/or longer loop-lengths. We now find an upper bound on the SUB for the CM-DM processing as

$$
\mathcal{R}_{i}^{(d), s u b}=\log _{2}\left(1+\Gamma^{-1} \sigma_{x i}^{2} \sigma_{v, i}^{2(d)} \mid h_{i}^{(c d)} \|^{2}\right) \leq \log _{2}\left(1+\Gamma^{-1} S_{i}^{(c d)}(N-1) \alpha^{2(c d)}\right),
$$

where $\mathbf{h}_{i}^{(c d)}$ denotes the $i$ th column of $\mathbf{H}^{(c d)}$, $S_{i}^{(c d)}=\frac{\sigma_{x, i}^{2}}{\sigma_{v^{\prime}, i}^{2(c d)}}$, and $\sigma_{v^{\prime}, i}^{2(c d)}=\frac{\sigma_{v, i}^{2(c d)}}{\left|H_{i i}^{(c d)}\right|^{2}}$ denote the crosstalkfree SNR and noise after FEQ for the CM-DM system, respectively.

The values of the rates achievable in these cases of concatenated CM-DM, the $N$-dimensional vectored

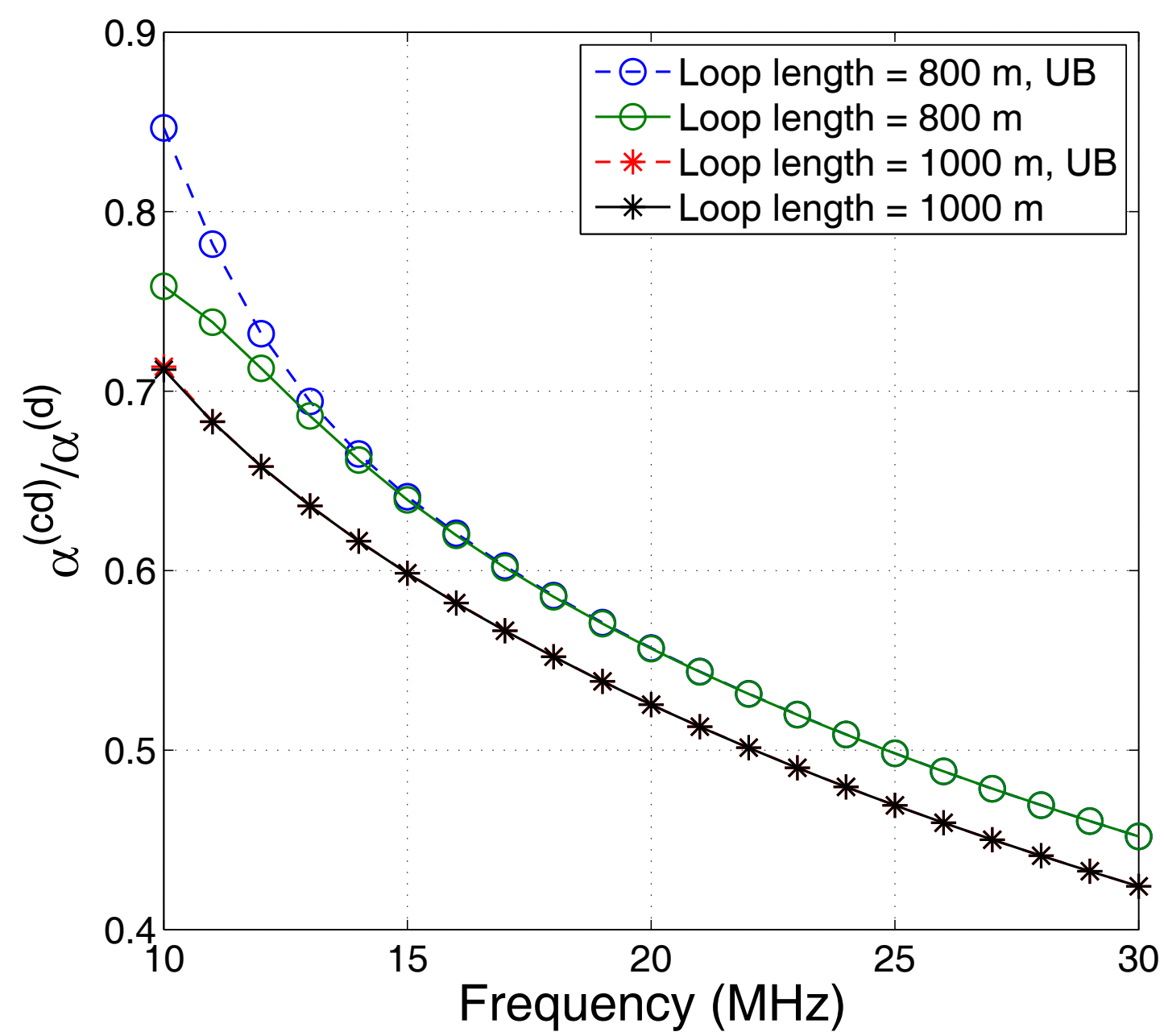

Figure 3 Ratio of CWDD parameters of $\mathrm{N}$-dimensional vectored CM-DM and DM channels versus frequency at loop lengths of $800 \mathrm{~m}$ and $1000 \mathrm{~m}$. 
CM-DM and the vectored DM, are taken up later in Section 7 , dealing with simulations and numerical results. It will be shown there that while the concatenated system produces the best results in all situations, the rate performance of the lower complexity $\mathrm{N}$-dimensional CM-DM processing is nearly identical to that of the concatenated system at larger frequencies or loop lengths. Next, we describe the proposed CM-DM processing with the ZF receiver in order to achieve the SUB.

\subsection{ZF receiver for CM-DM processing}

The ZF receiver (that performs close to the SUB) is a near optimal canceler for the vectored DM processing because of limited noise enhancement in well conditioned DSL channels [8]. Since CWDD property worsens with frequency and loop length, there may be a gap between the rate performance of $\mathrm{ZF}$ receiver and the SUB rate at higher frequency tones and/or longer looplengths. It is shown in the previous subsection that the $\mathrm{N}$-dimensional CM-DM processing offers superior CWDD characteristics than the corresponding DM processing, in particular at higher frequencies and/or longer loop-lengths. Hence, the $\mathrm{N}$-dimensional CM-DM processing can provide better rate performance for the crosstalk cancelation with the ZF receiver. On applying the ZF receiver to the $N$-dimensional CM-DM system, we obtain the data rate as

$$
\mathcal{R}_{i}^{(c d), \mathrm{zf}}=\log _{2}\left(1+\Gamma^{-1} \frac{S_{i}^{(c d)}}{\psi_{i}^{(c d), \mathrm{zf}}}\right),
$$

where $\psi_{i}^{(c d), z f}$ is the noise enhancement factor defined as [8]

$$
\psi_{i}^{(c d), \mathrm{zf}} \triangleq \frac{\left|\operatorname{det}\left(\mathbf{A}_{N-1}\right)\right|^{2}+(N-1)\left|\operatorname{det}\left(\mathbf{B}_{N-1}\right)\right|^{2}}{\left|\operatorname{det}\left(\mathbf{A}_{N}\right)\right|^{2}},
$$

and $\mathbf{A}_{N}$ denotes an $N \times N$ matrix whose diagonal elements are 1 and magnitude of non-diagonal elements is upper bounded by $\alpha^{(c d)}$. Also, the structure of the matrix $\mathbf{B}_{N}$ is same as that of $\mathbf{A}_{N}$ except that the magnitude of the last diagonal element is also upper bounded by $\alpha^{(c d)}$. The reader is referred to [8] for details. We now derive an upper bound on $\psi_{i}^{(c d), z \mathrm{z}}$ in the following Lemma 1 for the case of a ZF receiver applied on the vectored CM-DM combined signal.

Lemma 1 If $(N-1) \alpha^{(c d)}<1$, the noise enhancement factor of ZF receiver with CM-DM processing can be upper bounded by

$$
\psi_{i}^{(c d), 2 f} \leq \frac{\left(1+(N-2) \alpha^{2(c d d)}\right)^{N-1}+(N-1)^{2} \alpha^{2(c d d)}\left(1+(N-2) \alpha^{2(c d d)}\right)^{N-2}}{\left(1-(N-1) \alpha^{(c d)}\right)^{2 N}} .
$$

Proof By applying Hadamard's inequality as $\left|\operatorname{det}\left(\mathbf{A}_{N-1}\right)\right| \leq\left(\prod_{i=1}^{N-1} \sum_{j=1}^{N-1}\left|a_{i j}\right|^{2}\right)^{\frac{1}{2}}$, we can obtain upper bounds on $\left|\operatorname{det}\left(\mathbf{A}_{N-1}\right)\right|^{2}$ and $\left|\operatorname{det}\left(\mathbf{B}_{N-1}\right)\right|^{2}$ as follows:

$$
\begin{aligned}
& \left|\operatorname{det}\left(\mathbf{A}_{N-1}\right)\right|^{2} \leq\left(1+(N-2) \alpha^{2(c d)}\right)^{N-1}, \\
& \left|\operatorname{det}\left(\mathbf{B}_{N-1}\right)\right|^{2} \leq(N-1) \alpha^{2(c d)}\left(1+(N-2) \alpha^{2(c d)}\right)^{N-2} .
\end{aligned}
$$

Also by applying Ostrowski's inequality as $\left|\operatorname{det}\left(\mathbf{A}_{N}\right)\right| \geq \prod_{i=1}^{N}\left[\left|a_{i i}\right|-\sum_{j=1, j \neq i}^{N}\left|a_{i j}\right|\right]$, we can lower bound $\left|\operatorname{det}\left(\mathbf{A}_{N}\right)\right|^{2}$ as

$$
\left|\operatorname{det}\left(\mathbf{A}_{N}\right)\right| \geq\left(1-(N-1) \alpha^{(c d)}\right)^{N} .
$$

For the lower bound in (35) to hold, it is required that $\sum_{j=1, j \neq i}^{N}\left|a_{i j}\right|<1$, which in turn can be expressed as

$$
(N-1) \alpha^{(c d)}<1 .
$$

The condition $(N-1) \alpha^{(c d)}<1$ is readily satisfied for typical values of $N$ since $\alpha^{(c d)}$ normally takes a very small value. By substituting (34) and (35) in (32), we obtain

$$
\psi_{i}^{(c d), 2 f} \leq \frac{\left(1+(N-2) \alpha^{2(c d d)}\right)^{N-1}+(N-1)^{2} \alpha^{2(c d d)}\left(1+(N-2) \alpha^{2(c d d)}\right)^{N-2}}{\left(1-(N-1) \alpha^{(c d)}\right)^{2 N}} .
$$

The condition in (36) together with (37) completes the proof of Lemma 1.

It has been shown in [8] that noise enhancement factor of the ZF receiver for the DM system is close to unity due to the CWDD property of the DM channel. Since the $N$-dimensional CM-DM vectored channel possesses stronger CWDD characteristic than the DM channel, $\psi_{i}^{(c d), z \mathrm{zf}}$ is also close to unity. This implies that the ZF receiver can achieve performance close to the SUB of this lower-dimensional CM-DM system. Cendrillon et al. [8] have also presented an upper bound on the noise enhancement of the ZF canceler for the DM system. However, the upper bound in [8] is conditioned to exist for a limited number of users $(N \leq 25)$ and bandwidth (upto $12 \mathrm{MHz}$ ), whereas the upper bound in (33) is applicable with the condition $(N-1) \alpha^{(c d)}<1$, which is typical for a large number of users and frequencies. Hence, the derived bound presented here can also be used to predict accurately the performance of high-bandwidth VDSL2 (newest standard of xDSL) system. A lower bound on $\psi_{i}^{(c d), z f}$ is also presented in Appendix 1. 
The ZF receiver requires channel matrix inversion at each tone which causes an increase in its computational complexity. Such matrix inversions are required frequently due to changes in user status, or variations in crosstalk characteristics [14]. To avoid this, a low-order truncated series approximation of the inverse channel matrix was considered for the DM channel in the downstream transmissions [29]. We extend this concept for the vectored CM-DM processing in the upstream transmissions by applying a first order approximation of inverse of the combined CM-DM channel as

$$
\mathbf{H}^{(\mathbf{c d})^{-1}}=\left[\mathbf{H}_{\text {diag }}^{(c d)}\left(\mathbf{I}+\mathbf{C}^{(c d)}\right)\right]^{-1} \approx\left(\mathbf{I}-\mathbf{C}^{(c d)}\right) \mathbf{H}_{\text {diag }}^{(c d)^{-1}},
$$

where $\mathbf{H}_{\text {diag }}^{(c d)}=\operatorname{diag}\left(\mathbf{H}^{(c d)}\right)$ is a diagonal matrix of the CM-DM channel and $\mathbf{C}^{(c d)}$ is a zero-diagonal matrix whose $i j$ th element denotes the normalized FEXT coupling coefficients from $j$ th disturber to the $i$ th victim. Here, the normalization refers to the division of each matrix row by its associated diagonal entry. The point to be noted is that this algorithm, referred to as approximate ZF (AZF), does not involve inversion of matrix $\mathbf{H}$ ${ }^{(c d)}$ but requires the inversion of the diagonal matrix $\mathbf{H}_{\text {diag }}^{(c d)}$ only. By using the approximate inverse (38) as a canceler, we find the estimate of $i$ th symbol as

$$
\tilde{X}_{i}=X_{i}-\mathbf{C}^{2(c d)}(i,:) \mathbf{x}+\frac{\mathbf{v}^{(c d)}}{H_{i i}^{(c d)}}-\frac{\mathbf{C}^{(c d)}(i,:) \mathbf{v}^{(c d)}}{H_{i i}^{(c d)}} .
$$

By considering the worst case by assuming all nondiagonal elements of the matrix $\mathbf{C}^{(c d)}$ as $\alpha^{(c d)}$ and assuming equal transmit PSD and thermal noise, we simplify (39) to find an upper bound on noise enhancement of the AZF receiver for the $i$ th user as

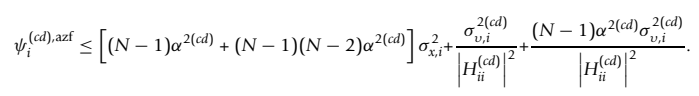

Note that with all combined CM-DM values replaced by DM channel values, (40) also holds for the vectored DM case. It can be seen from (40) that the AZF receiver can perform better for the CM-DM system than the DM signals at higher frequencies and/or longer looplengths (since $\alpha^{(c d)} \leq \alpha^{(d)}$ and $\left|H_{i i}^{(c d)}\right|^{2} \geq\left|H_{i i}^{(d)}\right|^{2}$ ). Thus, the rates of the ZF receiver with the vectored CM-DM processing will clearly be higher than those with DM processing alone, because the effective channel matrix after combining is more strongly CWDD in the former case. In general, it can be seen that the performance of the linear receivers can be improved with the vectored CM-DM processing.

\section{CM-DM processing for alien crosstalk cancelation}

In the absence of alien crosstalk, noise at different TPs is spatially white. This assumption was used in the analysis for CM-DM processing in the preceding sections. In the presence of the alien crosstalk originating from the external noise sources, noise among different TPs experiences a high spatial correlation. This fact has been emphasized in many publications $[3,10,30,31]$. Alien crosstalk can be particularly troublesome because it originates from other transmitters or channels that are not part of the system under design. Sources for alien crosstalk are, for example, RFI ingress, and signals from other DSL services. Cancelation of alien crosstalk is more involved and different techniques can be applied for its mitigation. One way is to exploit the spatial correlation by incorporating a whitening filter prior to applying cancelation algorithms. The presence of noise correlation clearly helps in mitigating the alien crosstalk. As such, a unity correlation (perfect correlation) completely eliminates the alien noise whereas there is no improvement if noise is uncorrelated. It is known that the non-white component of the noise (i.e., alien noise and crosstalk coupling from adjacent pairs) in the CM channel is correlated with the corresponding noise components in the DM [15]. In case of alien noise originating from a single or small number of noise sources, the correlation between DM and CM signals is strong. This high correlation can be used effectively to mitigate the alien noise in the DM channel. In what follows, we first extend the optimal linear combination of CM and DM signals at each TP in the presence of alien crosstalk. Later, we coordinate the single-TP CM-DM processing to obtain $\mathrm{N}$-dimensional CM-DM system and study its capacity.

\subsection{Single-TP CM-DM processing for alien crosstalk}

In the presence of alien crosstalk, $V_{i}^{(d)}$ and $V_{i}^{\prime(c)}$ of (12) also contains alien component $A_{i}^{(d)}$ and $A_{i}^{(c)}$, respectively. Therefore, we can write DM and CM signals after FEQ as

$$
\begin{aligned}
& Z_{a, i}^{(d)}=X_{i}+\sum_{j=1, j \neq i}^{N} \frac{H_{i j}^{(d)}}{H_{i i}^{(d)}} X_{j}+V_{a, i}^{(d)}, \\
& Z_{a, i}^{(c)}=X_{i}+\sum_{j=1, j \neq i}^{N} \frac{H_{i j}^{(c)}}{H_{i i}^{(c)}} X_{j}+V_{a, i}^{(c)},
\end{aligned}
$$

where $V_{a, i}^{\prime(d)}=\frac{V_{i}^{(d)}}{H_{i i}^{(d)}}+\frac{A_{i}^{(d)}}{H_{i i}^{(d)}}$ and $V_{a, i}^{(c)}=\frac{V_{i}^{(c)}}{H_{i i}^{(c)}}+\frac{A_{i}^{(c)}}{H_{i i}^{(c)}}$. The subscript " $a$ " is used to highlight that alien crosstalk has been considered. We denote the variance of $A_{i}^{(d)}$ and 
$\sigma_{a, i}^{(c)}$ by $\sigma_{a, i}^{(d)}$ and $\sigma_{a, i}^{(c)}$, respectively. Now, we can represent noise covariance matrix $\mathbf{R}_{a, i}$ of $\mathbf{z}_{a, i}^{(c d)}=\left[Z_{a, i}^{(d)}, Z_{a, i}^{(c)}\right]^{T}$ in terms of $\mathbf{R}_{i}$ of (14) as

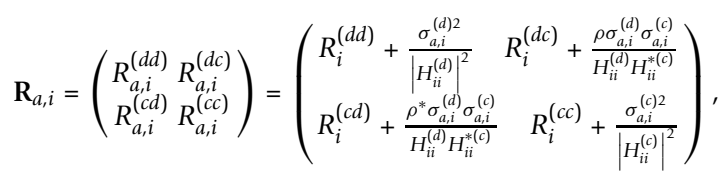

where $\rho$ is the correlation coefficient between CM and DM alien noise of the $i$ th TP. Similar to the white noise case, we combine CM and DM signals after FEQ in the presence of alien crosstalk to get $Z_{a, i}^{(c d)}=Z_{a, i}^{(d)}+K_{a, i} Z_{a, i}^{(c)}$. By applying an approach similar to that used in (18), we can obtain coefficient $K_{i, a}$ as

$$
K_{i, a}=\frac{\operatorname{SNR}_{a, i, f \times t}^{(c)}}{\operatorname{SNR}_{a, i, \text { fext }}^{(d)}}\left[\frac{1-\operatorname{SNR}_{a, i, \text { fext }}^{(d)}\left(\sum_{j \neq i} \frac{H_{i j}^{(d)} H_{i j}^{*(c)}}{H_{i j}^{(d)} H_{i i}^{*(c)}}-\frac{\rho \sigma_{i, a}^{(d)} \sigma_{i, a}^{(c)}}{\sigma_{x, i}^{2} H_{i i}^{(d)} H_{i i}^{*(c)}}\right)}{1-\operatorname{SNR}_{a, i, \text { fext }}^{(c)}\left(\sum_{j \neq i} \frac{H_{i j}^{*(d)} H_{i j}^{(c)}}{H_{i i}^{*(d)} H_{i i}^{(c)}}-\frac{\rho^{*} \sigma_{i, a}^{(d)} \sigma_{i, a}^{(c)}}{\sigma_{x, i}^{2} H_{i i}^{*(d)} H_{i i}^{(c)}}\right)}\right],
$$

From (17) and (43), it can be easily shown that $K_{i, a}=K_{i}$ for uncorrelated noise $(\rho=0)$. Similar to white noise, we can show that the linear combination approach maximizes the SNR in the presence of alien crosstalk.

\subsection{Capacity of the vectored CM-DM processing in the presence of alien crosstalk}

Similar to (27), we can coordinate signals by combining the received CM and DM signals using $K_{i, a}$ to obtain an $N$-dimensional CM-DM vectored system as

$$
\mathbf{y}_{a}^{(c d)}=\mathbf{H}^{(c d)} \mathbf{x}+\mathbf{v}_{a}^{(c d)}
$$

where $\quad \mathbf{y}_{a}^{(c d)}=\left[Y_{a, 1}^{(c d)}, Y_{a, 2}^{(c d)}, \ldots, Y_{a, N}^{(c d)}\right]^{T} \quad$ with $Y_{a, i}^{(c d)}=Y_{a, i}^{(d)}+K_{i} Y_{a, i}^{(c)}, \forall i$. It can be seen that $Y_{a, i}^{(d)}$ and $Y_{a, i}^{(c)}$ are received DM and CM signals at the $i$ th TP in the presence of alien noise. Similarly, $\mathbf{v}_{a}^{(c d)}=\left[V_{a, 1}^{(c d)}, V_{a, 2}^{(c d)}, \ldots, V_{a, N}^{(c d)}\right]$ with $V_{a, i}^{(c d)}=V_{a, i}^{(d)}+K_{i} V_{a, i}^{(c)}$, $\forall i$. In the presence of alien crosstalk, noise term $\mathbf{v}_{a}^{(c d)}$ is correlated among different TPs with a covariance matrix $W^{(c d)}$. This noise correlation can be exploited to mitigate alien noise. To do so, we whiten the noise vector $\mathbf{v}_{a}^{(c d)}$ by a whitening filter $\mathbf{W}^{-1}$ where $\mathbf{W}\left(=\mathbf{W}^{(c d) 1 / 2}\right)$ is a square root of the covariance matrix. In upstream transmissions, we can use the whitening filter $\mathbf{W}^{-1}$ prior to the application of an equalizer. The received $N$-dimensional signal $\mathbf{y}^{(c d)}$ of (44) can be processed by the whitening filter $\mathbf{W}^{-1}$ to yield

$$
\tilde{\mathbf{y}}^{(c d)}=\mathbf{W}^{-1} \mathbf{H}^{(\mathrm{cd})} \mathbf{X}+\mathbf{W}^{-1} \mathbf{v}_{\mathbf{a}}^{(\mathbf{c d})} .
$$

It can be seen that resultant noise $\mathbf{W}^{-1} \mathbf{v}_{\mathbf{a}}^{(\mathbf{c d})}$ is spatially uncorrelated with identity covariance matrix. We define an absolute operation $|\cdot|$ on a matrix $\mathbf{A}$ with elements $a_{i j}$ denoted by $|\mathbf{A}|$ whose $i j$ th element is $\left|a_{i j}\right|$. We now take absolute value of each element of the equivalent channel $\widetilde{\mathbf{H}}^{(c d)}=\mathbf{W}^{-1} \mathbf{H}^{(\mathrm{cd})}$ of (45) to get $\left|\widetilde{\mathbf{H}}^{(c d)}\right| \leq\left|\mathbf{W}^{-1}\right|\left|\mathbf{H}^{\text {cd }}\right|$, where the inequality is due to use of the relation $\left|\tilde{H}_{i j}^{(c d)}\right| \leq\left|\mathbf{W}^{-1}(i,:)\right|\left|\mathbf{H}^{(c d)}(:, j)\right|, \forall i, j$. We now use the definition of matrix inverse to find an upper bound on the $i$ th element of $\left|\mathbf{W}^{-1}\right|$ as $\left|\left[\mathbf{W}^{-1}\right]_{i i}\right| \leq \frac{\left|\operatorname{det}\left(\overline{\mathbf{W}}_{p}\right)\right|}{|\operatorname{det}(\mathbf{W})|} \quad \forall i$, and $\left|\left[\mathbf{W}^{-1}\right]_{i j}\right| \leq \frac{\left|\operatorname{det}\left(\overline{\mathbf{W}}_{n p}\right)\right|}{|\operatorname{det}(\mathbf{W})|} \quad \forall i \neq j$, where $\left|\operatorname{det}\left(\overline{\mathbf{W}}_{p}\right)\right|$ and $\left|\operatorname{det}\left(\overline{\mathbf{W}}_{n p}\right)\right|$ are, respectively, maximum absolute values of principal and non-principal minors (of sizes $N-1 \times N-1$ ) of the matrix $\mathbf{W}$. Using upper bounds on diagonal $\left|\left[\mathbf{W}^{-1}\right]_{i i}\right|$ and non-diagonal $\left|\left[\mathbf{W}^{-1}\right]_{i j}\right|$ elements of $\left|\mathbf{W}^{-1}(i,:)\right|$ in $\left|\widetilde{\mathbf{H}}^{(c d)}\right| \leq\left|\mathbf{W}^{-1}\right|\left|\mathbf{H}^{\text {cd }}\right|$, we can obtain an upper bound on the diagonal and non-diagonal elements of $\left|\widetilde{\mathbf{H}}^{(c d)}\right|$ as

$$
\begin{gathered}
\left|\widetilde{\mathbf{H}}_{i i}^{(c d)}\right| \leq \frac{\left|H_{i i}^{(c d)}\right|}{|\operatorname{det}(\mathbf{W})|}\left[\left|\operatorname{det}\left(\overline{\mathbf{W}}_{p}\right)\right|+(N-1) \alpha^{(c d)}\left|\operatorname{det}\left(\overline{\mathbf{W}}_{n p}\right)\right|\right] \quad \forall i, \\
\left|\widetilde{\mathbf{H}}_{j i}^{(c d)}\right| \leq \frac{\left|H_{i i}^{(c d)}\right|}{|\operatorname{det}(\mathbf{W})|}\left[\left|\operatorname{det}\left(\overline{\mathbf{W}}_{n p}\right)\right|+\alpha^{(c d)}\left|\operatorname{det}\left(\overline{\mathbf{W}}_{p}\right)\right|+(N-2) \alpha^{(c d)}\left|\operatorname{det}\left(\overline{\mathbf{W}}_{n p}\right)\right|\right] \forall j \neq i .
\end{gathered}
$$

Using (46) and (47), an upper bound on CWDD parameter of the modified channel $\widetilde{\mathbf{H}}^{(c d)}$ can be obtained as

$$
\widetilde{\alpha}^{(c d)} \leq \frac{\left|\operatorname{det}\left(\overline{\mathbf{W}}_{n p}\right)\right|+\alpha^{(c d)}\left|\operatorname{det}\left(\overline{\mathbf{W}}_{p}\right)\right|+(N-2) \alpha^{(c d)}\left|\operatorname{det}\left(\overline{\mathbf{W}}_{n p}\right)\right|}{\left|\operatorname{det}\left(\overline{\mathbf{W}}_{p}\right)\right|+(N-1) \alpha^{(c d)}\left|\operatorname{det}\left(\overline{\mathbf{W}}_{n p}\right)\right|} .
$$

We can obtain the SUB of $N$-dimensional CM-DM system in the presence of alien crosstalk as

$$
\widetilde{\mathcal{R}}_{i, a}^{(c d), \text { sub }} \leq \log _{2}\left(1+\Gamma^{-1} \widetilde{S}_{i, a}^{(c d)}\left(1+(N-1) \widetilde{\alpha}^{2(c d))}\right)\right)
$$

where self-crosstalk-free SNR $\widetilde{S}_{i, a}^{(c d)}$ after noise whitening can be upper bounded using (46) and $\widetilde{\sigma}_{v, a, i}^{2(c d)}=1$ as

$$
\tilde{S}_{i, a}^{(c d)} \leq \frac{\left|H_{i i}^{(c d)}\right|^{2} \sigma_{x, i}^{2}}{|\operatorname{det}(\mathbf{W})|^{2}}\left[\left|\operatorname{det}\left(\overline{\mathbf{W}}_{p}\right)\right|+(N-1) \alpha^{(c d)}\left|\operatorname{det}\left(\overline{\mathbf{W}}_{n p}\right)\right|\right]^{2}
$$

Since CM signals are highly correlated with the DM signals, $\widetilde{S}_{i, a}^{(c d)}$ for $N$-dimensional CM-DM system can be significantly higher than the DM counterpart. 
Consequently, the $\mathrm{N}$-dimensional CM-DM processing gives higher data rates than the DM processing. However, it is noted that the whitening procedure destroys the CWDD characteristic $\left(\widetilde{\alpha}^{(c d)}>>\alpha^{(c d)}\right)$ of DSL channels and thus the ZF receiver does not achieve the SUB. Non-linear receiver ZF-DFE [10] and SAGE based iterative receiver proposed in [9] are alternative candidates for the mitigation of alien crosstalk even with reduced CWDD characteristics of the DSL channels.

\section{Simulation and numerical results}

In this section, we investigate the performance of the vectored CM-DM processing using computer simulations and numerical results based on derived bounds. We consider Annex C band plan [1] which corresponds to three upstream frequency bands. We assume $10 \mathrm{TPs}$ of equal length in a binder while other simulation parameters as per the ITU-T standards [1] are listed in Table 1. We adopt the stochastic DM-channel model of [23] and use the proposed CM channel model in (6) for the simulations. We simulate the capacity of the proposed vectored CM-DM processing and compare with the DM processing in all the three upstream bands. For the white noise case, we demonstrate the rate performance of the ZF receiver for the $\mathrm{N}$-dimensional CMDM system model considered here. We also plot the data rates to verify the upper bound on the noise enhancement of ZF receiver in (33) for the $N$-dimensional CM-DM system in the absence of alien noise.

Figure 4 shows that concatenating the vectored $\mathrm{CM}$ and DM signals provides higher data-rate than the DM signals alone over a broad ranges of frequencies and loop lengths. This is consistent with the observations made by Magesacher et al. [4]. We also observe that when we combine the $\mathrm{CM}$ and DM signals optimally before vectoring, there is always a rate improvement, though its extent depends on the loop length and frequency. While the concatenated CM-DM performs best, as expected, at higher looplengths the performance of the $N$-dimensional CM-DM

Table 1 Simulation parameters

\begin{tabular}{cc}
\hline Twisted pairs & 24 AWG $(0.5 \mathrm{~mm})$ \\
Band plan & Annex C [1] \\
Profile & $30 \mathrm{a}$ \\
Tone width & $8.625 \mathrm{KHz}$ \\
Upstream transmit power & $+14.5 \mathrm{dBm}$ \\
Transmit signal PSD per tone & $-60 \mathrm{dBm} / \mathrm{Hz}$ \\
DM and CM Noise PSDs & $-130 \mathrm{dBm} / \mathrm{Hz}$ \\
Error probability & $10^{-7}$ \\
Coding gain & $3 \mathrm{~dB}$ \\
Noise margin & $6 \mathrm{~dB}$ \\
SNR gap $\Gamma$ & $12.8 \mathrm{~dB}$ \\
\hline
\end{tabular}

system seems to be nearly identical. (This is because the CM signal dominates at higher loop-lengths). It is clear that there is a large gap between the capacities achieved with combined $\mathrm{N}$-dimensional CM-DM and DM processing, which increases significantly with increase in the bandwidth and/or loop length. For lower frequencies and shorter loop lengths, however, the $\mathrm{N}$-dimensional CMDM processing achieves the same maximum data rate (SUB) as that of the DM processing. This is because the DM channel is much cleaner (less noisy) in this region. It can be further seen from Figure 4 that the ZF receiver achieves the SUB of the $N$-dimensional CM-DM processing. We also study the influence of the higher CM noise level on the capacity gain using the CM signals at high frequency tones in Figure 5. This figure depicts that there is a significant rate gain in the HFB using the $N$-dimensional CM-DM processing for longer loop-lengths even when the CM noise level is higher than the DM noise. Hence, in the absence of alien crosstalk, we can achieve higher datarates at longer loop-lengths.

To illustrate the effect of alien crosstalk, we consider noise correlation values of 0.99 and 0.50 and equal $\mathrm{CM}$ and $\mathrm{DM}$ alien power of $-100 \mathrm{dBm} / \mathrm{Hz}$. Figure 6 shows the capacity of the vectored $\mathrm{N}$-dimensional CM$D M$ and DM signals after whitening the correlated noise. An upper bound on the SUB (indicated in (49)) is also presented. It can be seen that the data-rate performance improves with increase in noise correlation of the alien crosstalk. It can also be observed that the $\mathrm{N}$-dimensional CM-DM processing is of much greater importance in the presence of alien crosstalk. As such, data rates of the $\mathrm{N}$-dimensional CM-DM is greater than that of DM with pronounced improvements at longer loop-lengths and higher frequencies as shown in Figure 6.

\section{Conclusion}

We have investigated the use of CM signals to increase the rate and reach of the VDSL systems in upstream transmissions. A vectored system consisting of both CM and DM signals has been proposed to enable the effective cancelation of crosstalk. We have shown that simply combining the CM and DM signals optimally results in rate improvement without significant increase in complexity. Further, vectoring such combined signals enables ZF type receivers to achieve the capacity due to the increased diagonal dominance of the resultant $\mathrm{N}$-dimensional CM-DM channel matrix. We have shown that the rate gain with CM-DM joint processing is a strong function of the loop length and frequency. In the presence of alien crosstalk, use of the CM signals has been shown to increase the data rate significantly. 


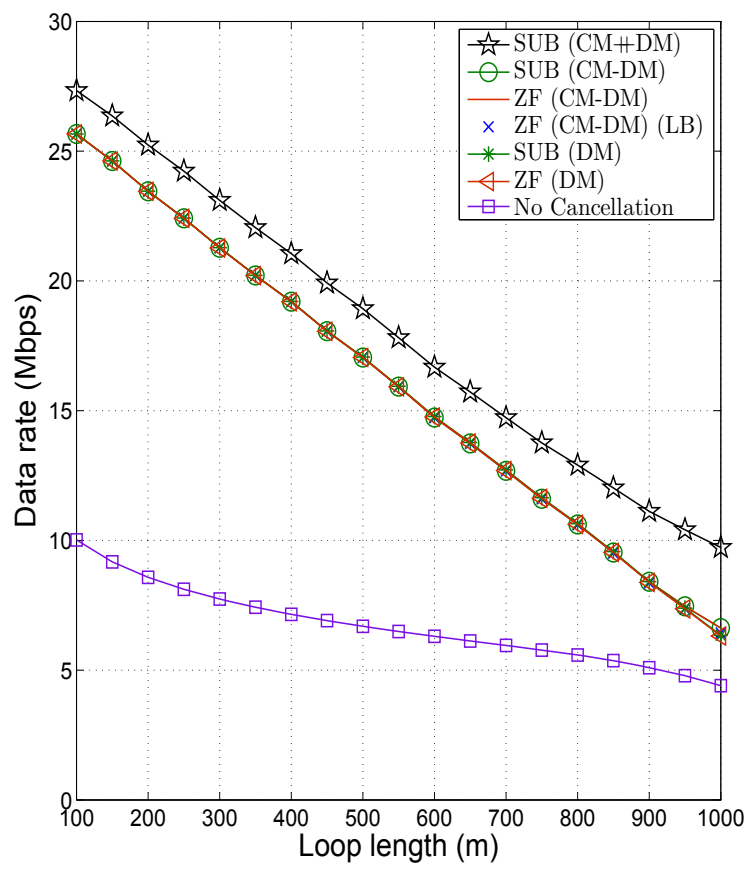

(a)

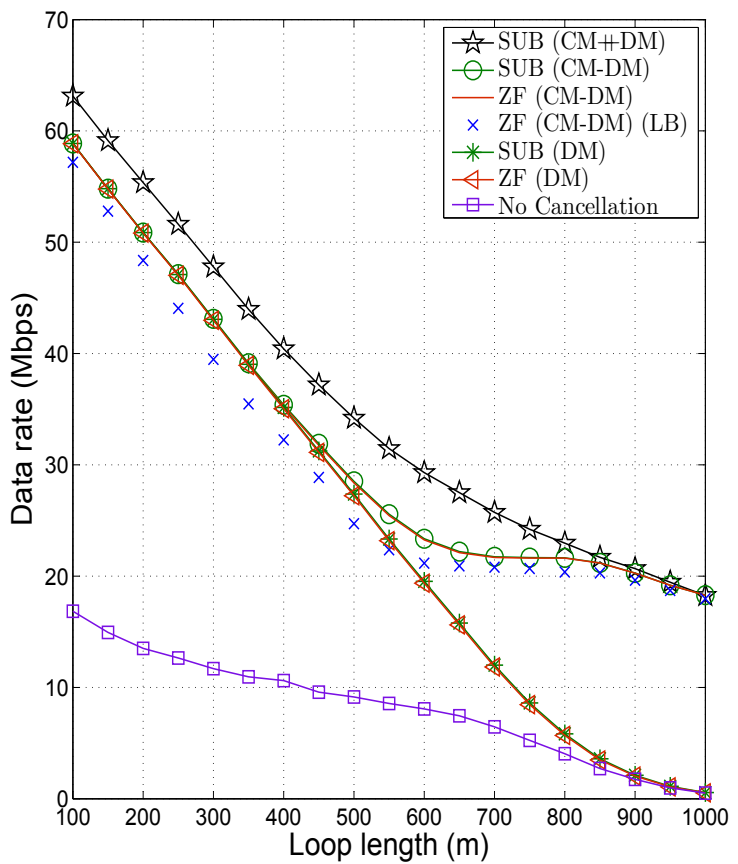

(b)

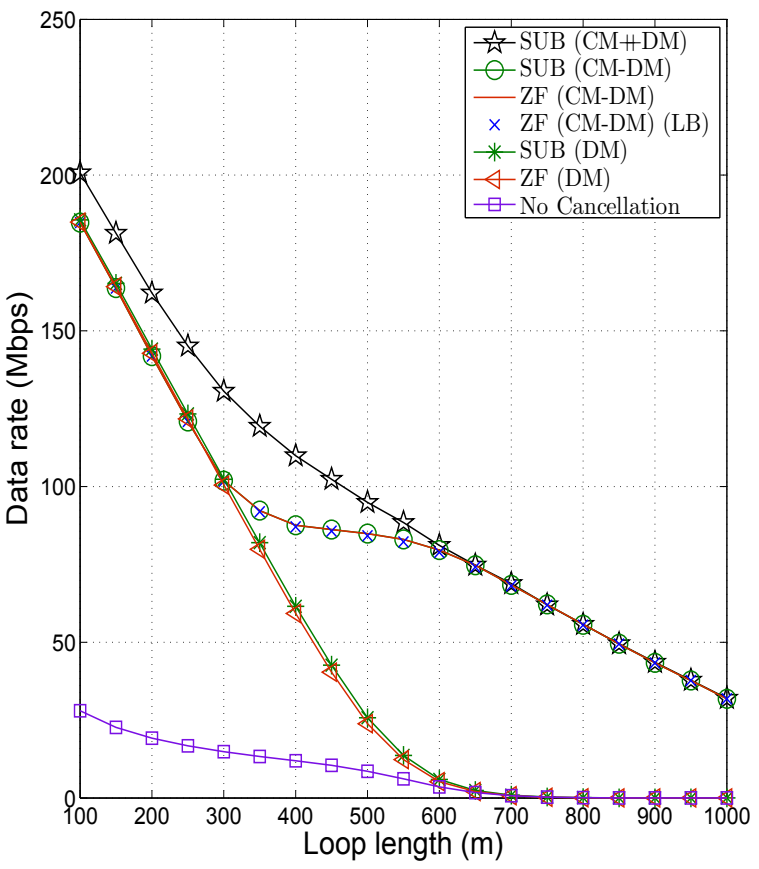

(c)

Figure 4 Data rate (SUB) performance versus loop length of the $\mathrm{N}$-dimensional vectored CM-DM and DM processing along with the ZF receiver for the three upstream bands. SUB with the concatenated (H) vectored CM and DM signals is also presented. 


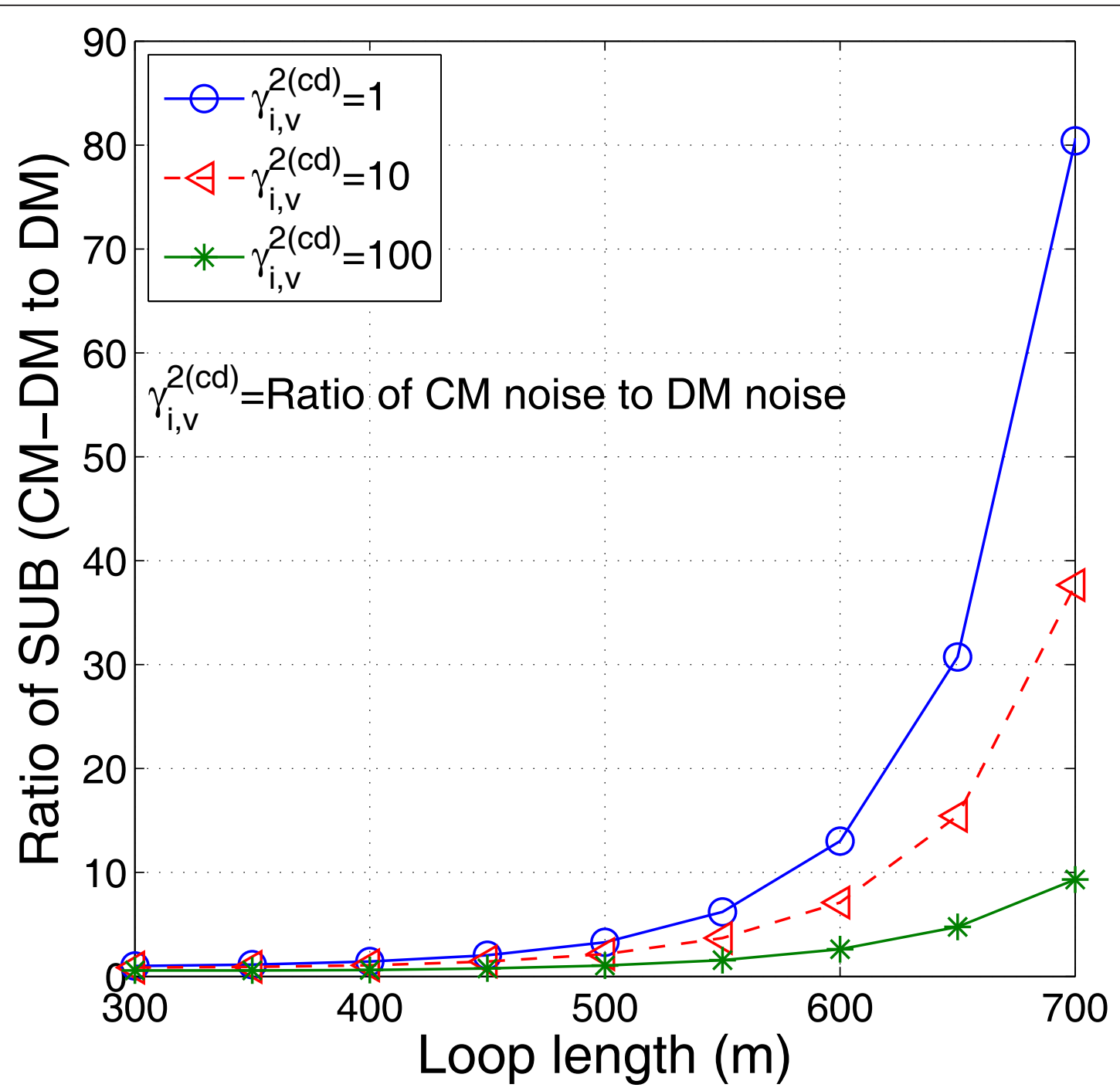

Figure 5 Effect of the CM noise on gain of data-rate (SUB) for the $\mathrm{N}$-dimensional vectored CM-DM processing in the high frequency band.

\section{Appendix 1: A lower bound on noise enhancement of zf receiver}

Similar to finding an upper bound, we use (32) to get a lower bound on $\psi_{i}^{(c d), z f}$. To facilitate this, we use Hadamard's inequality to find an upper bound on $\left|\operatorname{det}\left(\mathbf{A}_{N}\right)\right|^{2}$ as

$$
\left|\operatorname{det}\left(\mathbf{A}_{N}\right)\right|^{2} \leq\left(1+(N-1) \alpha^{2(c d)}\right)^{N-1} .
$$

If $(N-2) \alpha^{(c d)}<1$, Ostrowski's inequality is applied to obtain a lower bound on $\left|\operatorname{det}\left(\mathbf{A}_{N-1}\right)\right|^{2}$ as

$$
\left|\operatorname{det}\left(\mathbf{A}_{N-1}\right)\right|^{2} \geq\left(1-(N-2) \alpha^{(c d)}\right)^{2(N-2)} .
$$

We can use absolute value of minimum and maximum eigenvalues $\left(\lambda_{b}^{l}, \lambda_{b}^{u}\right)$ of the matrix $\mathbf{B}_{N-} 1$ to find a lower bound on $\left|\operatorname{det} \mathbf{B}_{N-1}\right|^{2}$ as [32],

$$
\left|\operatorname{det}\left(\mathbf{B}_{N-1}\right)\right|^{2} \geq\left(\lambda_{b}^{l}\right)^{2 \Omega_{b}}\left(\lambda_{b}^{u}\right)^{2\left(N-1-\Omega_{b}\right)},
$$

where

$$
\Omega_{b}=(N-1)\left[\frac{\lambda_{b}^{u}-\mu_{\lambda_{b}}}{\lambda_{b}^{u}-\lambda_{b}^{l}}\right] .
$$

Absolute values of mean $\mu_{\lambda_{b}}=\frac{\operatorname{tr}\left(\mathbf{B}_{N-1}\right)}{N-1}$ and variance $\sigma_{\lambda_{b}}^{2}$ of eigenvalues of $\mathbf{B}_{N-1}$ are given as 


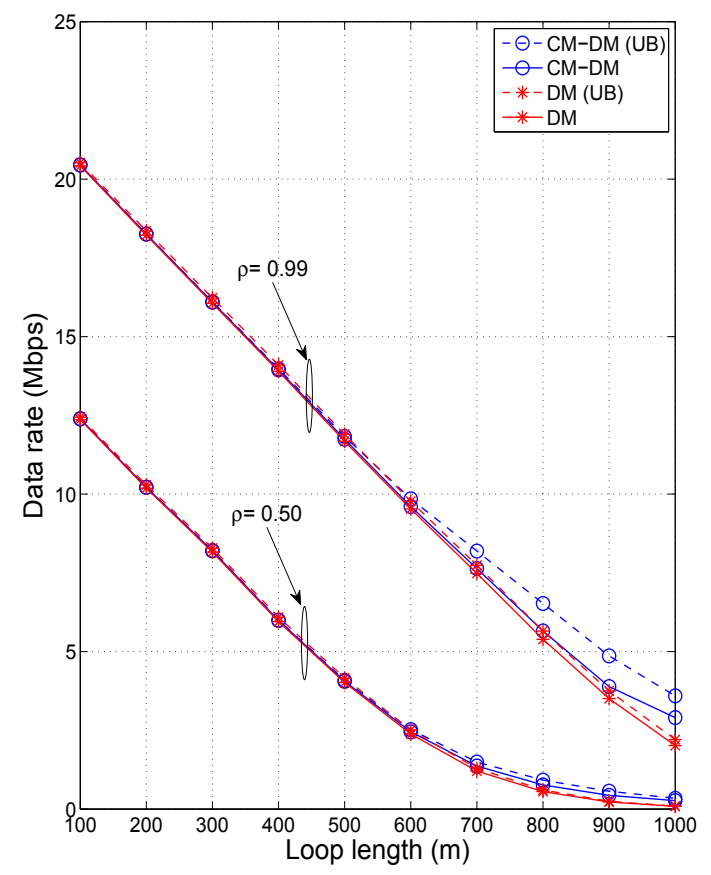

(a)

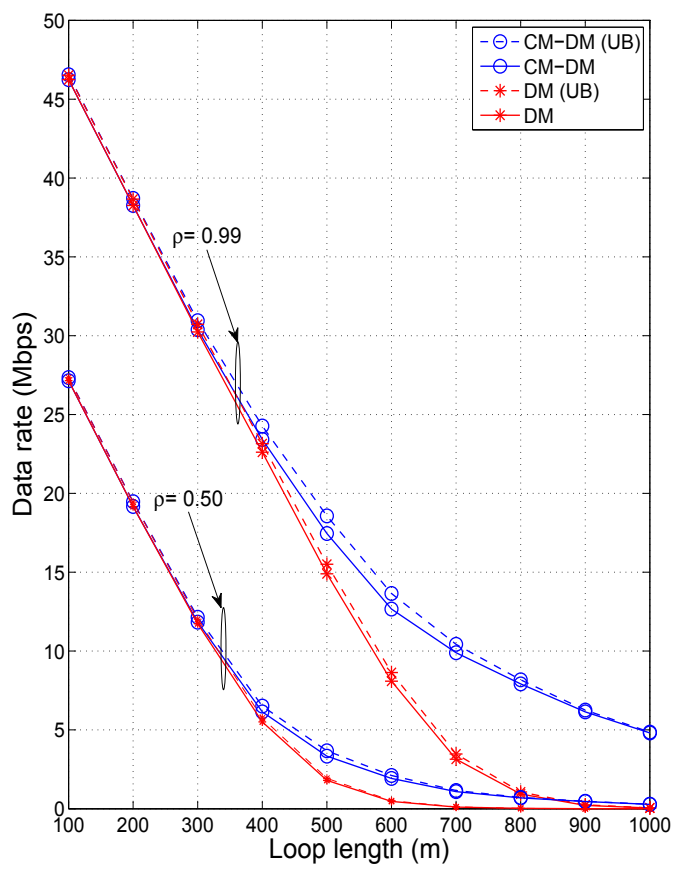

(b)

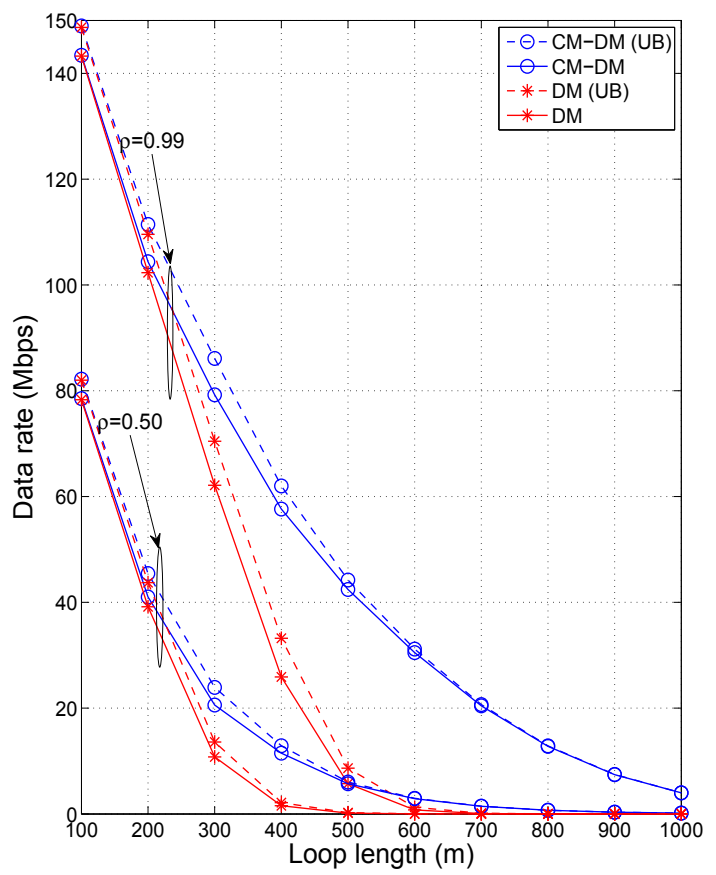

(c)

Figure 6 Data rate (SUB) performance of the $N$-dimensional vectored CM-DM and DM processing versus loop length in the presence of alien crosstalk for the three upstream bands. Alien crosstalk correlation coefficient of 0.99 and 0.50 and alien power of $-100 \mathrm{dBm} / \mathrm{Hz}$ (for both the CM and DM systems) are considered. 


$$
\begin{aligned}
& \frac{N-2}{N-1} \leq\left|\mu_{\lambda_{b}}\right| \leq 1+\frac{\alpha^{(c d)}-1}{N-1} \\
& \sigma_{\lambda_{b}}^{2}=\frac{\operatorname{tr}\left(\mathbf{B}_{N-1}^{\dagger} \mathbf{B}_{N-1}\right)}{N-1}-\left|\mu_{\lambda_{b}}\right|^{2} \leq \frac{(N-1)\left(N^{2}-3 N+3\right) \alpha^{2(c d)}+(N-2)}{(N-1)^{2}} .
\end{aligned}
$$

We now use the result of [33] to find magnitude of minimum eigenvalue of the matrix $\mathbf{B}_{N-1}$ as

$$
\lambda_{b}^{l}=\mu_{\lambda_{b}}-\sigma_{\lambda_{b}}(N-2)^{1 / 2} .
$$

Substituting (55) and (56) in (57), we get

$$
\lambda_{b}^{l}=\frac{N-2}{N-1}-\sqrt{\frac{(N-2)}{(N-1)^{2}}\left((N-1)\left(N^{2}-3 N+3\right) \alpha^{2(c d)}+(N-2)\right)} .
$$

We use Geršgorin theorem [34] to find magnitude of maximum eigenvalue of the matrix $\mathbf{B}_{N-1}$ as

$$
\lambda_{b}^{u}=(N-2) \alpha^{(c d)}+1 .
$$

We substitute (54), (58), and (59) in (53) to obtain a lower bound on $\left|\operatorname{det} \mathbf{B}_{N-1}\right|^{2}$. Then substituting (51), (52), and (53) in (32), a lower bound on $\psi_{i}^{(c d), z f}$ can be obtained.

\section{List of abbreviations}

VDSL: Very high-speed digital subscriber lines; CM: common mode; DM: differential mode; MIMO: muti-input multi-output; TP: twisted pair; ZF: zero forcing; SUB: single user bound; CO: central office; CPE: customer premise equipment; DMT: discrete multi tone; DFE: decision feedback equalizer; SAGE: space alternating generalized expectation maximization; MMSE: minimum mean square error; RFI: radio frequency interference; SNR: signalto-noise ratio; CWDD: column wise diagonally dominant; LFB: low-frequency band; MFB: mid-frequency band; HFB: high-frequency band; FEQ: frequencydomain equalization; FEXT: far end crosstalk; PSD: power spectral density; ML: maximum likelihood; MOE: minimum output energy; AZF: approximate zero forcing; ITU-T: international telecommunication uniontelecommunication (standardization sector).

\section{Acknowledgements}

This study was supported by research grants from Conexant Systems Inc. USA, and IIT Delhi. An earlier preliminary version of this study consisting of discussion on the CM channel model was presented at the Annual IEEE Indicon Conference, INDICON 2008, IIT Kanpur, India, in December 2008. The authors would like to thank the anonymous reviewer for useful comments which helped to improve the quality of the article.

\section{Competing interests}

The authors declare that they have no competing interests.

Received: 9 March 2011 Accepted: 12 March 2012

Published: 12 March 2012

\section{References}

1. ITU-T Recommendation G.993.2 (G.VDSL2), Very high speed digita subscriber line transceivers 2 (VDSL2). International Telecommunication Union Telecommunication Standardization Sector (ITU-T), (Febreuary 2006)

2. T Starr, M Sorbara, JM Cioffi, P Silverman, DSL Advances (Prentice-Hall, Upper Saddle River, NJ, USA, 2003)

3. G Ginis, C-N Peng, Alien crosstalk cancellation for multipair digital subscriber line systems. EURASIP Journal on Applied Signal Processing. 2006 12 (2006). Article ID 16828
4. T Magesacher, P Ödling, PO Börjesson, W Henkel, T Nordström, R Zukunft, $S$ Haar, On the capacity of the copper cable channel using the common moden, in Proceedings of the IEEE Global Telecommunications Conference (GLOBECOM '02), (2) (Taipei, Taiwan, November 2002), pp. 1269-1273

5. S Jagannathan, V Pourahmad, K Seong, JM Cioffi, M Ouzzif, R Tarafi, Common-mode data transmission using the binder sheath in digital subscriber lines. IEEE Trans Commun. 57(3), 831-840 (2009)

6. B Lee, JM Cioffi, S Jagannathan, K Seong, Y Kim, M Mohseni, MH Brady, Binder MIMO channels. IEEE Trans Commun. 55(8), 1617-1628 (2007)

7. G Ginis, JM Cioffi, Vectored transmission for digital subscriber line systems. IEEE J Sel Areas Commun. 20(5), 1085-1104 (2002). doi:10.1109/ JSAC.2002.1007389

8. R Cendrillon, G Ginis, E Van den Bogaert, M Moonen, A near-optimal linear crosstalk canceler for upstream VDSL. IEEE Trans Signal Process. 54(8), 3136-3146 (2006)

9. SM Zafaruddin, S Prakriya, S Prasad, Iterative receiver based on SAGE algorithm for crosstalk cancellation in upstream vectored VDSL. ISRN Communications and Networking. 2011, 15 (2011). Article ID 586574

10. Chiang-Yu Chen, K Seong, R Zhang, JM Cioffi, Optimized resource allocation for upstream vectored DSL systems with zero-forcing generalized decision feedback equalizer. IEEE J Sel Top Signal Process. 1(4), 686-699 (2007)

11. PK Pandey, M Moonen, L Deneire, MMSE-based partial crosstalk cancellation for upstream VDSL, in Proceedings of IEEE International Conference on Communications (ICC'10), (1) (Cape Town, South Africa, May 2010), pp. 1-5

12. I Wahibi, M Ouzzif, JL Masson, S Saoudi, Crosstalk cancellation in upstream coordinated DSL using an iterative MMSE receiver, in Proceedings of IEEE International Conference on Communications (ICC'09), (1) (Cape Town, Germany, June 2009), pp. 1-5

13. R Cendrillon, M Moonen, G Ginis, KV Acker, T Bostoen, P Vandaele, Partial crosstalk cancellation for upstream VDSL. EURASIP J Appl Signal Process. 2004(10), 1520-1535 (2004). doi:10.1155/\$1110865704309273

14. AR Forouzan, LM Garth, Computationally efficient partial crosstalk cancellation in fast time-varying DSL crosstalk environments. EURASIP Journal on Advances in Signal Processing. 2007, 15 (2007). Article ID 72041

15. T Magesacher, W Henkel, T Nordström, P Ödling, PO Börjesson, On the correlation between common-mode and differential-mode signals, in Temporary Document TD 45, 013t45 ETSI STC TM6, (1) (Stockholm, Sweden, September 2001), pp. 1-5

16. P Ödling, PO Börjesson, T Magesacher, T Nordströom, An approach to analog mitigation of RFI. IEEE J Sel Areas Commun. 20(5), 974-986 (2002). doi:10.1109/JSAC.2002.1007379

17. TH Yeap, DK Fenton, PD Lefebvre, A novel common-mode noise cancellation technique for VDSL applications. IEEE Trans Instrum Meas. 52(4), 1325-1334 (2003). doi:10.1109/TIM.2003.816850

18. AH Kamkar-Parsi, M Bouchard, G Bessens, TH Yeap, A wideband crosstalk canceller for xDSL using common-mode information. IEEE Trans Commun. 53(2), 238-242 (2005). doi:10.1109/TCOMM.2004.841981

19. L Pierrugues, P Duvaut, J Pons, A Santraine, SIMO-NAR NEXT canceller for DMT DSL-CPE: a stochastic shannon capacity perspective, in Proceedings of the IEEE Sarnoff Symposium (Sarnoff'06), (1) (Princeton, NJ, USA, March 2006), pp. 1-6

20. T Magesacher, P Ödling, PO Börjesson, Analysis of adaptive interference cancellation using common-mode information in wireline communications. EURASIP J Adv Signal Process. 2007, 11 (2007). Article ID 84956

21. T Magesacher, P Ödling, PO Börjesson, T Nordström, Exploiting the common-mode signal in XDSL, in Proceedings of the European Signal Processing Conference (EU-SICPCO'04), (1) (Vienna, Austria, September 2004), pp. 1217-1220

22. T Magesacher, P Ödling, PO Börjesson, S Shamai (Shitz), Information rate bounds in common-mode aided wireline communications. Eur Trans Telecommun. 17(5), 533-545 (2006). doi:10.1002/ett.1071

23. M Sorbara, P Duvaut, F Shmulyian, S Singh, A Mahadevan, Construction of a DSL-MIMO channel model for evaluation of FEXT cancellation systems in VDSL2, in Proceedings of the IEEE Sarnoff Symposium (Sarnoff'07), (1) (Princeton, NJ, USA, 2007), pp. 1-6

24. J Maes, M Guenach, M Peeters, Statistical MIMO channel model for gain quantification of DSL crosstalk mitigation techniques, in Proceedings of IEEE International Conference on Communications (ICC'09), (1) (Dresden, Germany, June 2009), pp. 1-5

25. W Xu, C Schroeder, PA Hoeher, A stochastic MIMO model for far-end crosstalk in VDSL cable binders, in Proceedings of IEEE International 
Conference on Communications (ICC'09), (1) (Dresden, Germany, June 2009), pp. 1-5

26. M Jakovljević, T Magesacher, K Ericson, P Ödling, PO Börjesson, S Zazo,

Common mode characterization and channel model verification for shielded twisted pair (STP) cable, in Proceedings of the IEEE International Conference on Communications (ICC'08), (1) (Beijing, China, May 2008), pp. 447-451

27. M Jakovljević, T Magesacher, P Ödling, PO Börjesson, M Sanchez, S Zazo, Throughput of shielded twisted-pair cables using wire-shield modes in the presence of radio ingress, in Proceedings of 16th International Conference on Digital Signal Processing (DSP'09), (1) (Santorini, Greece, July 2009), pp. 1-6

28. T Starr, JM Cioffi, PJ Silverman, Understanding Digital Subscriber Line Technology Prentice Hall, Englewood Cliffs, NJ, USA, (1999)

29. A Leshem, L Youming, A low complexity linear precoding technique for next generation VDSL downstream transmission over copper. IEEE Trans Signal Process. 55(11), 5527-5534 (2007)

30. JM Cioffi, S Jagannathan, G Ginis, M Brady, DSM ppstream data rate revival: the restoration of symmetry. AT/S ContrbutioNIPP-NAl, 2006-092, (Savannah, 26 June 2006), pp. 1-9

31. L Sandstrom, KW Schneider, LL Joiner, A Wilson, Spatial correlation of alien crosstalk in MIMO DSL systems. IEEE Trans Commun. 57(8), 2269-2271 (2009)

32. B Kalantari, TH Pate, A determinantal lower bound. Linear Algebra Appl. 326, 151-159 (2001). doi:10.1016/50024-3795(00)00287-1

33. SH Wolkowicz, GPH Styan, Bounds for eigenvalues using traces. Linear Algebra Appl. 29, 471-506 (1980)

34. RA Horn, CR Johnson, Matrix Analysis (Cambridge University Press, Cambridge, UK, 1985)

doi:10.1186/1687-6180-2012-63

Cite this article as: Zafaruddin et al.: Performance analysis of a common-mode signal based low-complexity crosstalk cancelation scheme in vectored VDSL. EURASIP Journal on Advances in Signal Processing 2012 2012:63.

\section{Submit your manuscript to a SpringerOpen ${ }^{\mathcal{O}}$ journal and benefit from:}

- Convenient online submission

- Rigorous peer review

- Immediate publication on acceptance

- Open access: articles freely available online

- High visibility within the field

- Retaining the copyright to your article

Submit your next manuscript at $\gg$ springeropen.com 